\title{
Presupernova evolution of accreting white dwarfs with rotation
}

\author{
S.-C. Yoon and N. Langer
}

Astronomical Institute, Utrecht University, Princetonplein 5, 3584 CC, Utrecht, The Netherlands

Received 8 December 2003 / Accepted 26 January 2004

\begin{abstract}
We discuss the effects of rotation on the evolution of accreting carbon-oxygen white dwarfs, with the emphasis on possible consequences in Type Ia supernova (SN Ia) progenitors. Starting with a slowly rotating white dwarf, we consider the accretion of matter and angular momentum from a quasi-Keplerian accretion disk. Numerical simulations with initial white dwarf masses of $0.8,0.9$ and $1.0 M_{\odot}$ and accretion of carbon-oxygen rich matter at rates of $3 \ldots 10 \times 10^{-7} M_{\odot} / \mathrm{yr}$ are performed. The models are evolved either up to a ratio of rotational to potential energy of $T / W=0.18-$ as angular momentum loss through gravitational wave radiation will become important for $T / W<0.18-$ or to central carbon ignition. The role of the various rotationally induced hydrodynamic instabilities for the transport of angular momentum inside the white dwarf is investigated. We find that the dynamical shear instability is the most important one in the highly degenerate core, while Eddington-Sweet circulations, Goldreich-Schubert-Fricke instability and secular shear instability are most relevant in the nondegenerate envelope. Our results imply that accreting white dwarfs rotate differentially throughout, with a shear rate close to the threshold value for the onset of the dynamical shear instability. As the latter depends on the temperature of the white dwarf, the thermal evolution of the white dwarf core is found to be relevant for the angular momentum redistribution. As found previously, significant rotation is shown to lead to carbon ignition masses well above $1.4 M_{\odot}$. Our models suggest a wide range of white dwarf explosion masses, which could be responsible for some aspects of the diversity observed in SNe Ia. We analyze the potential role of the bar-mode and the $r$-mode instability in rapidly rotating white dwarfs, which may impose angular momentum loss by gravitational wave radiation. We discuss the consequences of the resulting spin-down for the fate of the white dwarf, and the possibility to detect the emitted gravitational waves at frequencies of $0.1 \ldots 1.0 \mathrm{~Hz}$ in nearby galaxies with LISA. Possible implications of fast and differentially rotating white dwarf cores for the flame propagation in exploding white dwarfs are also briefly discussed.
\end{abstract}

Key words. stars: white dwarfs - stars: rotation - supernovae: general - gravitational waves - accretion, accretion disks

\section{Introduction}

Type Ia Supernovae (SNe Ia) have a particular importance in astrophysics. Observations of SNe Ia at low redshift showed a clear correlation between the peak brightness and the width of the light curve (Phillips 1993; Phillips' relation), which made it possible to use $\mathrm{SNe}$ Ia as distance indicators for galaxies even beyond $z=1$. This made SNe Ia an indispensable tool for cosmology, in particular for determining the cosmological parameters (e.g. Hamuy et al. 1996; Branch 1998; Leibundgut 2000, 2001). The new cosmology with a non-zero cosmological constant has been initiated by the observational evidence from SNe Ia at high redshift (Perlmutter et al. 1999; Riess et al. 2000).

Recent analyses of $\mathrm{SNe}$ Ia have revealed, however, that $\mathrm{SNe}$ Ia are not perfectly homogeneous but show some diversity in their light curves and spectra (e.g. Branch 2001; Nomoto et al. 2003; Li et al. 2003). This leaves concerns about applying Phillips' relation to very distant SNe Ia. An understanding of the origin of the diversity observed in SNe Ia is thus crucial for

Send offprint requests to: S.-C. Yoon, e-mail: s.c.Yoon@astro.uu.nl stellar evolution theory, which requires identifying the detailed evolutionary paths of SNe Ia progenitors.

Unlike core collapse supernovae, Type Ia supernovae (SNe Ia) are believed to occur exclusively in binary systems (e.g. Livio 2001). Although it is still unclear which kinds of binary systems lead to SNe Ia, non-degenerate stars such as main sequence stars, red giants or helium stars are often assumed as companion to a white dwarf (e.g. Li \& van den Heuvel 1997; Hachisu et al. 1999; Langer et al. 2000; Yoon \& Langer 2003). The white dwarf is then assumed to grow to the Chandrasekhar limit by mass accretion from its companion, with accretion rates which allow steady shell hydrogen and helium burning $\left(\dot{M} \gtrsim 10^{-7} M_{\odot} /\right.$ yr). An understanding of the physics of mass accretion is therefore indispensable for investigating the evolution of accreting white dwarfs.

Although the mass accretion process in white dwarfs has been discussed by many authors (e.g. Iben 1982; Nomoto 1982; Fujimoto \& Sugimoto 1982; Saio \& Nomoto 1985, 1998; Kawai et al. 1988; Cassisi et al. 1998; Piersanti et al. 2000; Langer et al. 2002), little attention has so far been devoted to the effects of angular momentum accretion and the ensuing white dwarf rotation (see Sect. 3.1). As the evolution of stars can generally be affected by rotation (e.g. 
Heger \& Langer 2000; Maeder \& Meynet 2000), this might be particularly so in accreting white dwarfs: since the transferred matter from the companion stars may form a Keplerian disk which carries a large amount of angular momentum, the resultant accretion of angular momentum will lead to the spinup of the white dwarf (e.g. Durisen 1977; Ritter 1985; Narayan \& Popham 1989; Langer et al. 2000, 2002, 2003). The observation that white dwarfs in cataclysmic variables rotate much faster than isolated ones (Sion 1999) provides evidence for accreting white dwarfs indeed being spun up. Rapidly rotating progenitors may also lead to aspherical explosions, which may give rise to the observed polarization of SNe Ia (Howell et al. 2001; Wang et al. 2003).

Here, we make an attempt to investigate in detail the possibility of angular momentum accretion, and the role of the various rotationally induced hydrodynamic instabilities in transporting angular momentum into the white dwarf core, and in establishing the pre-explosion angular momentum profile. The remainder of this paper is organized as follows. We evaluate the possible mechanisms for angular momentum transport in accreting white dwarfs in Sect. 2. Our approach to the problem, including the numerical methods and physical assumptions, is discussed in Sect. 3, where previous papers on rotating white dwarf models are also reviewed (Sect. 3.1). Numerical results are presented in Sect. 4, with the emphasis on the process of angular momentum transport in the white dwarf interior. Preexplosion conditions of accreting white dwarfs and their implications for the diversity of SNe Ia are discussed in Sects. 5, 6 and 7 . The possibility of detecting gravitational waves from $\mathrm{SNe}$ Ia progenitors is examined in Sect. 8. Our conclusions are summarized in Sect. 9.

\section{Angular momentum transport in white dwarfs}

Inside a white dwarf, angular momentum can be transported by Eddington-Sweet circulations and by turbulent diffusion induced by hydrodynamic instabilities. Our discussion below is limited to angular momentum transport in the vertical direction.

\subsection{Eddington-Sweet circulation}

Eddington-Sweet circulations are induced by the thermal imbalance between the equator and the poles of a rotating star. Its time scale is roughly given by $\tau_{\mathrm{ES}} \simeq \tau_{\mathrm{KH}} / \chi^{2}$ (Maeder \& Meynet 2000), where $\tau_{\mathrm{KH}}$ is the Kelvin-Helmholtz time scale and $\chi$ is the angular velocity normalized to the Keplerian value, i.e., $\chi=\omega / \omega_{\text {Kep }}$. In a white dwarf that accretes at rates $\dot{M}>10^{-7} M_{\odot} / \mathrm{yr}$, the surface luminosity reaches $10^{4} L_{\odot}$ due to compressional heating and nuclear burning. The quantity $\chi$ is close to 1 in the outer envelope as we shall see in Sect. 4.3.5. As a result, we expect an Eddington-Sweet circulation time scale in the non-degenerate envelope which is shorter than or comparable to the accretion time scale:

$$
\begin{aligned}
\tau_{\mathrm{ES}} & \approx \frac{G \Delta M_{\mathrm{env}}^{2}}{R L \chi^{2}} \\
& \approx 1.3 \times 10^{3} \mathrm{yr} \frac{\left(\Delta M_{\mathrm{env}} / 0.05 M_{\odot}\right)^{2}}{\left(R / 0.01 R_{\odot}\right)\left(L / 10^{4} L_{\odot}\right)(\chi / 0.8)^{2}} .
\end{aligned}
$$

In the degenerate core, on the other hand, we have $\tau_{\mathrm{ES}} \gg \tau_{\text {acc }}$ since the Kelvin-Helmholtz time scale is typically much larger than $10^{7}$ yr. Furthermore, $\chi$ is usually significantly smaller than 1 in the inner core:

$$
\begin{aligned}
\tau_{\mathrm{ES}} & \approx \frac{G M_{\mathrm{core}}^{2}}{R_{\mathrm{core}} L_{\mathrm{core}} \chi^{2}} \\
& \approx 7 \times 10^{9} \mathrm{yr} \frac{\left(M_{\mathrm{core}} / M_{\odot}\right)^{2}}{\left(R_{\mathrm{core}} / 0.005 R_{\odot}\right)\left(L_{\mathrm{core}} / 10 L_{\odot}\right)(\chi / 0.3)^{2}} .
\end{aligned}
$$

Consequently, Eddington-Sweet circulations are only important in the white dwarf envelope.

\subsection{Shear instability}

The dynamical shear instability (DSI) occurs when the energy of the shear motion dominates over the buoyancy potential. In a rotating flow, the linear condition for instability is given by

$\frac{N^{2}}{\sigma^{2}}<R_{\mathrm{i}, \mathrm{c}} \approx 1 / 4$

where $R_{\mathrm{i}, \mathrm{c}}$ denotes the critical Richardson number which is about $0.25, N^{2}$ the Brunt-Väisälä frequency, and $\sigma$ the shear factor (Zahn 1974):

$N^{2}=N_{\mathrm{T}}^{2}+N_{\mu}^{2}$

$N_{\mathrm{T}}^{2}=\frac{g \delta}{H_{P}}\left(\nabla_{\mathrm{ad}}-\nabla\right), \quad N_{\mu}^{2}=\frac{g \varphi \nabla_{\mu}}{H_{P}}$

and

$\sigma=\frac{\partial \omega}{\partial \ln r}$.

Here, $\omega$ is the angular velocity and the other symbols have their usual meaning. The equation of state of electron degenerate matter is given by $P=P\left(\rho, T, \mu_{\mathrm{e}}, \mu_{\mathrm{I}}\right)$ and the factor $\varphi \nabla_{\mu}$ in Eq. (5) needs to be replaced by $\varphi_{\mathrm{e}} \nabla_{\mu_{\mathrm{e}}}+\varphi_{\mathrm{I}} \nabla_{\mu_{\mathrm{I}}}$, where $\varphi_{\mathrm{e}}=\left(\partial \ln \rho / \partial \ln \mu_{\mathrm{e}}\right)_{P, T, \mu_{\mathrm{I}}}$ and $\varphi_{\mathrm{I}}=\left(\partial \ln \rho / \partial \ln \mu_{\mathrm{I}}\right)_{P, T, \mu_{\mathrm{e}}}$. In a typical CO white dwarf, $N_{\mu}^{2}$ is generally smaller than $N_{\mathrm{T}}^{2}$ and has minor effects on the angular momentum transport as discussed in Sect. 4.3.4.

With constant temperature and homogeneous chemical composition, which is a good approximation for the central region of a $\mathrm{CO}$ white dwarf, the critical value of $\sigma$ for the DSI can be given as

$$
\begin{aligned}
\sigma_{\text {DSI,crit }}^{2} & =N^{2} / R_{\mathrm{i}, \mathrm{c}} \\
& \simeq 0.2\left(\frac{g}{10^{9} \mathrm{~cm} / \mathrm{s}^{2}}\right)\left(\frac{\delta}{0.01}\right)\left(\frac{H_{P}}{8 \times 10^{7} \mathrm{~cm}}\right)^{-1}\left(\frac{\nabla_{\mathrm{ad}}}{0.4}\right)
\end{aligned}
$$

where $R_{\mathrm{i}, \mathrm{c}}=0.25$ is assumed. Figure 1 shows $\left|\sigma_{\mathrm{DSI}, \text { crit }}\right|$ as a function of density, with other physical assumptions as indicated in the figure caption. This figure shows that the condition for the DSI becomes more relaxed with increasing density, which is due to the buoyancy force becoming weaker with stronger degeneracy. This implies that the highly degenerate core in a white dwarf is more susceptible to the dynamical shear instability than the outer envelope. 


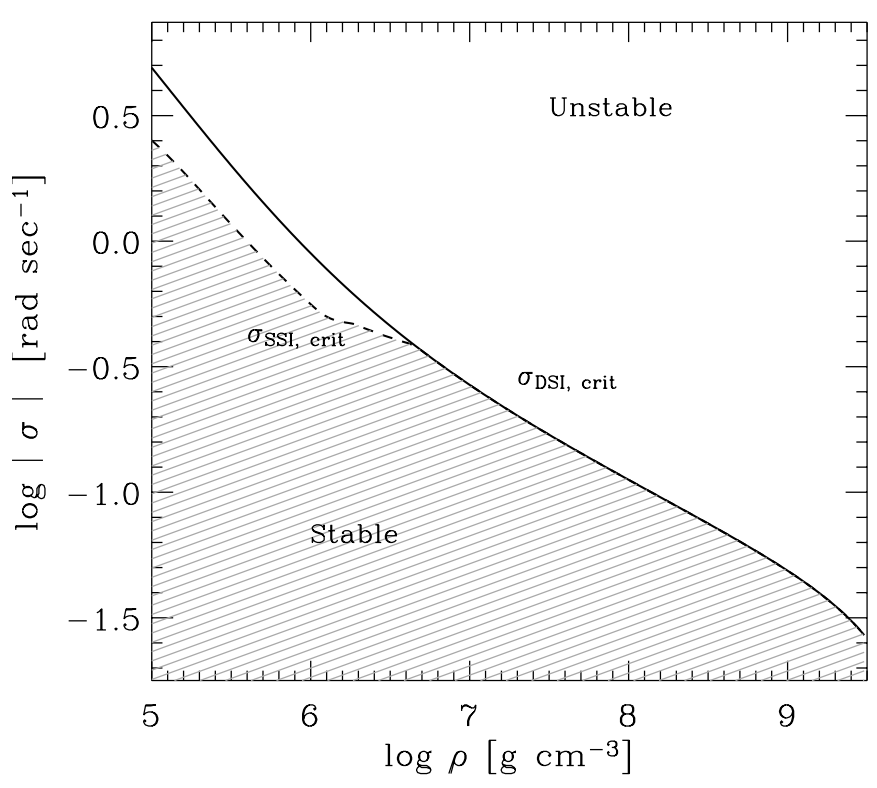

Fig. 1. Threshold values of the shear factor $\sigma=(\partial \omega / \partial \ln r)$ for the dynamical (solid line) and the secular (dashed line) shear instability. Constant gravity and temperature, i.e., $g=10^{9} \mathrm{~cm} / \mathrm{s}^{2}, T=5 \times 10^{7} \mathrm{~K}$, are assumed. The chemical composition is also assumed to be constant, with $X_{\mathrm{C}}=0.43$ and $X_{\mathrm{O}}=0.54$. For the critical Richardson number, $R_{\mathrm{i}, \mathrm{c}}=1 / 4$ is employed. $R_{\mathrm{e}, \mathrm{c}}=2500$ is used for the critical Reynolds number.

The stability criterion for the shear instability can be relaxed if thermal diffusion reduces the buoyancy force such that

$R_{\mathrm{is}, 1}:=\frac{v R_{\mathrm{e}, \mathrm{c}} N_{\mathrm{T}}^{2}}{K \sigma^{2}}<R_{\mathrm{i}, \mathrm{c}}$,

where $R_{\mathrm{e}, \mathrm{c}}$ is the critical Reynolds number, $v$ is the kinetic viscosity and $K=\left(4 a c T^{3}\right) /\left(3 C_{\mathrm{p}} \kappa \rho^{2}\right)$ is the thermal diffusivity (e.g. Maeder $\&$ Meynet 2000). Since the $\mu$ gradient is not affected by the thermal diffusion, this condition needs to be supplemented by the following additional condition (Endal \& Sofia 1976):

$R_{\mathrm{is}, 2}:=\frac{N_{\mu}^{2}}{\sigma^{2}}<R_{\mathrm{i}, \mathrm{c}}$.

The thermally induced shear instability is often called "secular shear instability" (SSI).

This instability can occur only when the thermal diffusion time scale is shorter than the turbulent viscous time scale (i.e., $v R_{\mathrm{e}, \mathrm{c}}<K$ ), which is often the case in non-degenerate stars. However, this condition is not always fulfilled in white dwarfs. In Fig. 2, the critical density $\rho_{\text {SSI,crit }}$ above which the secular shear instability is not allowed is plotted as a function of temperature. In this calculation, the ion and electron viscosity as well as the radiative and conductive opacities are estimated as described in Sect. 3.2. This figure indicates that the SSI may play a role in only relatively weakly degenerate regions.

The threshold value of the shear factor for this instability is given by $\sigma_{\mathrm{SSI}, \text { crit }}^{2}=v R_{\mathrm{e}, \mathrm{c}} N_{\mathrm{T}}^{2} /\left(K R_{\mathrm{i}, \mathrm{c}}\right)$ if $N_{\mu}^{2}=0$, and plotted in Fig. 1, with the assumptions as indicated in the figure caption. As shown in the figure and as discussed above, thermal diffusion cannot weaken the restoring buoyancy force for

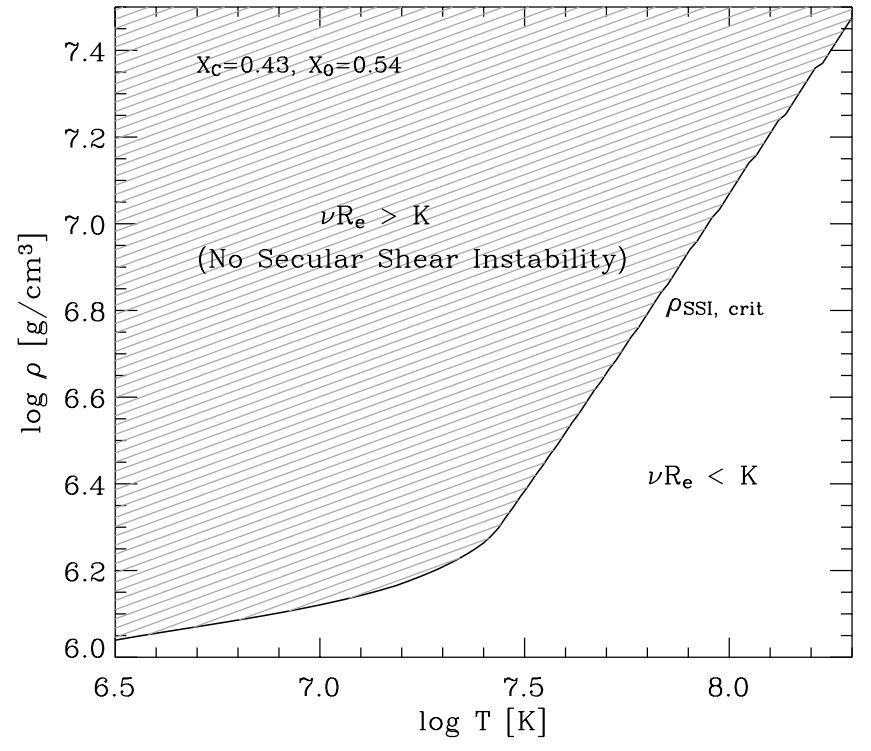

Fig. 2. The critical density $\rho_{\text {SSI,crit, }}$ above which the secular shear instability cannot occur, as function of temperature (solid line). The region where the thermal diffusion time scale exceeds the turbulent viscous time scale is hatched. Abundances of $X_{\mathrm{C}}=0.43$ and $X_{\mathrm{O}}=0.54$ are assumed. $R_{\mathrm{e}, \mathrm{c}}=2500$ is used for the critical Reynolds number.

$\rho \gtrsim 5 \times 10^{6} \mathrm{~g} / \mathrm{cm}^{3}$ under the chosen conditions, and only the dynamical shear instability can occur for higher densities.

Zahn (1992) derives the diffusion coefficient for the SSI as

$D_{\mathrm{SSI}}=\frac{1}{3} \frac{K \sigma^{2} R_{\mathrm{i}, \mathrm{c}}}{N^{2}}$

The time scale of the SSI when $\sigma=\sigma_{\text {DSI,crit }}$ thus becomes simply the thermal diffusion time scale: $\tau_{\text {SSI }}$ (at $\sigma=$ $\left.\sigma_{\text {DSI,crit }}\right) \approx 3 R^{2} / K$. In the degenerate core of a $\mathrm{CO}$ white dwarf with $\rho \lesssim \rho_{\text {SSI,crit }}$, we have $\tau_{\text {SSI }} \approx 7.6 \times 10^{6}\left(R_{\text {core }} / 2 \times\right.$ $\left.10^{8} \mathrm{~cm}\right)^{2} /\left(K / 500 \mathrm{~cm}^{2} \mathrm{~s}^{-1}\right) \mathrm{yr}$.

As a consequence of these considerations, any shear motion with $\sigma>\sigma_{\text {DSI,crit }}$ will decay such that $\sigma$ approaches $\sigma_{\text {DSI,crit }}$ on the dynamical time scale. Further angular momentum transport will operate on the thermal diffusion time scale until $\sigma$ reaches $\sigma_{\text {SSI,crit }}$ for $\rho \lesssim \rho_{\text {SSI,crit. }}$ For $\sigma<\sigma_{\text {SSI,crit }}$ and $\sigma<\sigma_{\text {DSI,crit }}$ (hatched region in Fig. 1), angular momentum will be transported only via the electron viscosity or via Eddington-Sweet circulations on much longer time scales (unless other kinds of instabilities are invoked). Therefore, if we consider accretion at rates required by the single degenerate $\mathrm{SN}$ Ia progenitor scenario $\left(\dot{M}>10^{-7} M_{\odot} / \mathrm{yr}\right)$, the degree of shear may not be far from the threshold value for the dynamical shear instability throughout the degenerate white dwarf interior. This conjecture is confirmed by the numerical results presented in Sect. 4.2.

\subsection{GSF and magnetic instabilities}

The Goldreich, Schubert and Fricke instability (GSF instability) can be induced if a star is in a baroclinic condition (Goldreich \& Schubert 1967; Fricke 1968). In an accreting white dwarf, this instability may be important in the nondegenerate envelope, but is likely suppressed in the degenerate 
core where the barotropic condition will be retained through a dynamical meridional circulation (Kippenhahn \& Möllenhoff 1974; see also Durisen 1977). Magnetic instabilities such as the Tayler instability (Spruit 2002) are potentially important, and their role will be investigated in the near future. In the present study, we restrict our discussions to non-magnetized white dwarfs.

\section{Simulations of accreting white dwarfs}

For the detailed investigation of the angular momentum transport and its role in the evolution of accreting white dwarfs, we have simulated accreting $\mathrm{CO}$ white dwarfs with various initial masses and accretion rates.

\subsection{Previous work on rotating white dwarfs}

To compare our results with previously obtained ones, here we briefly discuss the models of rotating white dwarfs in the literature. Most rotating white dwarf equilibrium models have been constructed assuming a barotropic equation of state and axial symmetry for both rigidly and differentially rotating cases (e.g. James 1964; Roxburgh 1965; Monaghan 1966; Ostriker 1966; Anand 1968; Ostriker \& Mark 1968; Hachisu 1986). These models have mainly been used to investigate the stability of rapidly rotating zero temperature white dwarfs (e.g. James 1964; Lynden-Bell \& Ostriker 1967; Ostriker \& Bodenheimer 1968; Durisen 1975b; Durisen \& Imammura 1981). One of the main results of these studies is that the Chandrasekhar limit increases slightly in rigidly rotating white dwarfs $\left(\sim 1.48 M_{\odot}\right)$, while differentially rotating white dwarfs can be dynamically stable even with masses up to $\sim 4.0 M_{\odot}$. It was also found that differentially rotating white dwarfs become secularly unstable to gravitational wave radiation if the ratio of the rotational energy to the gravitational energy exceeds a certain limit (see Sect. 5 for more detailed discussions).

The evolution of rotating white dwarfs was investigated by Durisen (1973a,b, 1975a) for the first time. Evolution and spinup of mass-accreting white dwarfs were probed by Durisen (1977). In his studies based on two-dimensional barotropic differentially rotating white dwarf models, Durisen assumed that angular momentum in the white dwarf interior is only transported by electron viscosity, and angular momentum transport time scales of the order of $10^{10} \mathrm{yr}$ have been obtained. In all these models, the thermal history and the energy transport in the white dwarf matter were neglected, assuming zero temperature.

Recently, Piersanti et al. (2003) investigated the effect of rotation on the thermal evolution of CO-accreting white dwarfs, using a one dimensional stellar evolution code. They also discussed angular momentum loss by gravitational wave radiation (cf. Sect. 5). However, the detailed history of the angular momentum transport inside the white dwarf was neglected, but rigid body rotation was assumed. Thus, while Durisen assumed much slower angular momentum redistribution by restricting his considerations to electron diffusion, Piersanti et al. assume instant angular momentum redistribution with a maximum efficiency.
Uenishi et al. (2003) considered a finite spin-up time of accreting white dwarfs by dividing their two-dimensional white dwarf models into a non-rotating core and a fast rotating outer envelope. However, rigid body rotation is again assumed for the spun-up region, introducing a discontinuity in the angular velocity profile.

In the present study on the evolution of accreting white dwarfs, we consider the effects of the accretion-induced heating and energy transport, the angular momentum transport by various instabilities, and the effect of rotation on the white dwarf structure. However, our numerical models are calculated in a one dimensional approximation as described in the next section, and thus the structure of rapidly rotating white dwarfs is described less accurately than in multi-dimensional models mentioned above. Furthermore, by assuming $\mathrm{CO}$-accretion we do not consider effects of nuclear shell burning. These, and the feedback between rotation and nuclear shell burning, as well as rotationally induced chemical mixing, are discussed in a separate paper (Yoon et al. 2004). The effects of rotation with respect to the off-center helium detonation models - i.e., helium-accreting $\mathrm{CO}$ white dwarf models with $\dot{M}=$ $\sim 10^{-8} M_{\odot} / \mathrm{yr}-$ are investigated in Yoon \& Langer (2004).

\subsection{Numerical method}

We use a hydrodynamic stellar evolution code (Langer et al. 1988) for the accreting white dwarf model calculations. Radiative opacities are taken from Iglesias \& Rogers (1996). For the electron conductive opacity, we follow Hubbard \& Lampe (1969) in the non-relativistic case, and Canuto (1970) in the relativistic case. The accreted matter is assumed to have the same entropy as that of the surface of the accreting white dwarf, and the accretion induced compressional heating is treated as in Neo et al. (1977).

The effect of rotation on the stellar structure is approximated in one dimension by introducing the factors $f_{\mathrm{P}}$ and $f_{\mathrm{T}}$ in the momentum and energy conservation equations (cf. Heger et al. 2000), following the method of Kippenhahn \& Thomas (1970) and Endal \& Sofia (1976). This method is well suited for the case where the effective gravity can be derived from an effective potential. This is indeed a good assumption for a $\mathrm{CO}$ white dwarf, since it should be in a barotropic condition except for the non-degenerate outer envelope. Note that this method is also applicable in the case of shellular rotation as discussed by Meynet \& Maeder (1997).

However, our method of computing the effective gravitational potential in a rotating star limits the accuracy of our results for very rapid rotation. The potential is expanded in terms of spherical harmonics, of which we only consider terms up to the second order (cf., Kippenhahn \& Thomas 1970). Fliegner (1993) showed this method to accurately reproduce the shapes of rigidly rotating polytropes up to a rotation rate of about $60 \%$ critical, corresponding to correction factors of $f_{\mathrm{P}} \simeq 0.75$ and $f_{\mathrm{T}} \simeq 0.95$ in the stellar structure equations (cf. Heger et al. 2000). We therefore limit these factors to the quoted values, with the consequence that we underestimate the effect of the centrifugal force in layers which rotate faster than 
about $60 \%$ critical. As in our models the layers near the surface are always close to critical rotation, the stellar radius of our models may be underestimated. Furthermore, our models are per definition rotationally symmetric. Therefore, we are in principle unable to investigate the onset of triaxial instabilities, which may affect the final fate of a rapidly rotating white dwarf (Sect. 5).

The angular momentum transport induced by the instabilities mentioned in Sect. 2 is described as a diffusion process (Heger et al. 2000), while this approximation neglects the possibility of advective angular momentum redistribution by Eddington-Sweet circulations (Maeder \& Meynet 2000). Note also that this approach is based on the assumption of shellular rotation (see Heger et al. 2000 for a detailed discussion), which might not be appropriate for white dwarfs. Nevertheless, our models can represent the case of cylindrical rotation to some degree, since most of the total angular momentum is confined to layers near the equatorial plane in both cases. Since the dynamical shear instability is important in the present study, the diffusion solver has been improved as a non-linear process, as explained in Yoon (2004), in order to deal properly with such a fast angular momentum redistribution which occurs on a dynamical time scale during the secular evolution of accreting white dwarfs. Diffusion coefficients for each of the instabilities are taken from Heger et al. (2000) and Heger \& Langer (2000), with some modifications as follows.

As mentioned in Sect. 2.3, the GSF instability may be suppressed in the degenerate barotropic core, and we describe this effect as

$D_{\mathrm{GSF}}=D_{\mathrm{GSF}}^{*}\left(1-\min \left[1, \frac{P_{\mathrm{e}}}{P_{\text {ideal }}}\right]\right)$.

Here, $D_{\mathrm{GSF}}^{*}$ is the diffusion coefficient for the GSF instability as estimated in Heger et al. (2000). $P_{\mathrm{e}}$ is the electron pressure for complete non-relativistic degeneracy, and $P_{\text {ideal }}$ is the ideal gas pressure:

$P_{\mathrm{e}}=9.91 \times 10^{12}\left(\frac{\rho}{\mu_{\mathrm{e}}}\right)^{5 / 3}, \quad P_{\text {ideal }}=\frac{\rho k T}{\mu m_{\mathrm{H}}}$.

For the secular instability, Zahn's (1992) prescription is employed (Eq. (10)), with a correction factor as in Heger et al. (2000) to ensure a smooth turn-on of the instability and to relate its strength to the deviation from the Richardson criterion:

$D_{\mathrm{SSI}}=\frac{1}{3} \frac{K \sigma^{2} R_{\mathrm{i}, \mathrm{c}}}{N^{2}}\left(1-\frac{\max \left\{R_{\mathrm{is}, 1}, R_{\mathrm{is}, 2}\right\}}{R_{\mathrm{i}, \mathrm{c}}}\right)^{2}$,

where $R_{\mathrm{is}, 1}$ and $R_{\mathrm{is}, 2}$ are defined in Eqs. (8) and (9). As already discussed in Sect. 2.2, the viscosity is an important factor for the stability of the secular shear instability. The former way of computing the ion viscosity in Heger et al. (2000) and Heger \& Langer (2000), following Spitzer (1962), is not well adapted for matter in the ion liquid state: here we follow the work of Wallenborn \& Bauss (1978), as described in Itoh et al. (1987):

$\eta_{\mathrm{i}}=5.53 \times 10^{3} \mathrm{ZA}^{-1 / 3}\left(\rho / 10^{6}\right)^{5 / 6} \eta^{*}\left(\mathrm{~g} \mathrm{~cm}^{-1} \mathrm{~s}^{-1}\right)$.
Table 1. Physical quantities of the initial white dwarf models: mass, surface luminosity, central temperature, central density, radius and rotation velocity.

\begin{tabular}{cccccc}
\hline \hline $\begin{array}{c}M_{\text {WD,init }} \\
M_{\odot}\end{array}$ & $\begin{array}{c}L_{\text {s,init }} \\
L_{\odot}\end{array}$ & $\begin{array}{c}T_{\text {c,init }} \\
10^{8} \mathrm{~K}\end{array}$ & $\begin{array}{c}\rho_{\text {c,init }} \\
10^{7} \mathrm{~g} / \mathrm{cm}^{3}\end{array}$ & $\begin{array}{c}R_{\text {WD,init }} \\
R_{\odot}\end{array}$ & $\begin{array}{c}v_{\text {rot,init }} \\
\mathrm{km} \mathrm{s}^{-1}\end{array}$ \\
\hline 0.8 & 0.80 & 0.79 & 0.97 & 0.0115 & 10 \\
0.9 & 0.88 & 0.85 & 1.68 & 0.0100 & 10 \\
1.0 & 0.81 & 0.94 & 3.14 & 0.0086 & 10 \\
\hline
\end{tabular}

We constructed a fitting formula for the dimensionless parameter $\eta^{*}$ from the table in Itoh et al. as

$$
\begin{aligned}
\eta^{*}= & -0.016321227+1.0198850 \Gamma^{-1.9217970} \\
& +0.024113535 \Gamma^{0.49999098}
\end{aligned}
$$

where $\Gamma=(Z e)^{2} /\left[\left(3 / 4 \pi n_{\mathrm{i}}\right)^{1 / 3} k T\right]$. The electron viscosity, which is dominant over the ion viscosity in degenerate matter, is estimated according to Nandkumar \& Pethick (1984).

When differential rotation is present in a star, rotational energy is dissipated through frictional heating. We estimate the rotational energy dissipation rate as (Kippenhahn \& Thomas 1978; Mochkovitch \& Livio 1989)

$\epsilon_{\mathrm{diss}}=\frac{1}{2} v_{\mathrm{turb}} \sigma^{2} \quad\left(\operatorname{erg~g}^{-1} \mathrm{~s}^{-1}\right)$,

where $v_{\text {turb }}$ is the turbulent viscosity, which is the sum of all the diffusion coefficients related to the rotationally induced instabilities considered in the present study (Heger et al. 2000).

As discussed in Sect. 2.2, the calculation of $\varphi_{\mu_{\mathrm{e}}}$ and $\varphi_{\mu_{\mathrm{I}}}$ is required for considering chemical composition effects in degenerate matter on the rotationally induced instabilities. We can make use of the thermodynamic relation:

$\varphi_{\mathrm{e}} \nabla_{\mu_{\mathrm{e}}}+\varphi_{\mathrm{I}} \nabla_{\mu_{\mathrm{I}}}=\frac{\mathrm{d} \ln \rho}{\mathrm{d} \ln P}-\alpha+\delta \nabla$,

where all the thermodynamic quantities on the right hand side are obtained from the equation of state used in our stellar evolution code (Blinnikov et al. 1996).

\subsection{Physical assumptions}

Our $\mathrm{CO}$ white dwarf models start mass accretion at three different initial masses: $0.8,0.9$ and $1.0 M_{\odot}$. Isolated white dwarfs are observationally found to rotate slowly ( $v_{\mathrm{WD}} \lesssim 40 \mathrm{~km} \mathrm{~s}^{-1}$; Heber et al. 1997; Koester et al. 1998; Kawaler 2003), as also predicted by stellar evolution models (Langer et al. 1999). We thus consider slow rigid rotation in our initial models, and the surface velocity at the white dwarf equator is set to $10 \mathrm{~km} \mathrm{~s}^{-1}$. Other physical quantities of the initial models are summarized in Table 1.

For the accretion rate four different values are considered: $3 \times 10^{-7}, 5 \times 10^{-7}, 7 \times 10^{-7}$ and $10^{-6} M_{\odot} / \mathrm{yr}$. These accretion rates are chosen in the context of the single degenerate Chandrasekhar mass scenario for SNe Ia progenitors, in which steady nuclear shell burning of hydrogen or helium is assumed (Nomoto 1982; Li \& van den Heuvel 1997; Langer et al. 2000; Yoon \& Langer 2003). The accreted matter is carbon-oxygen 


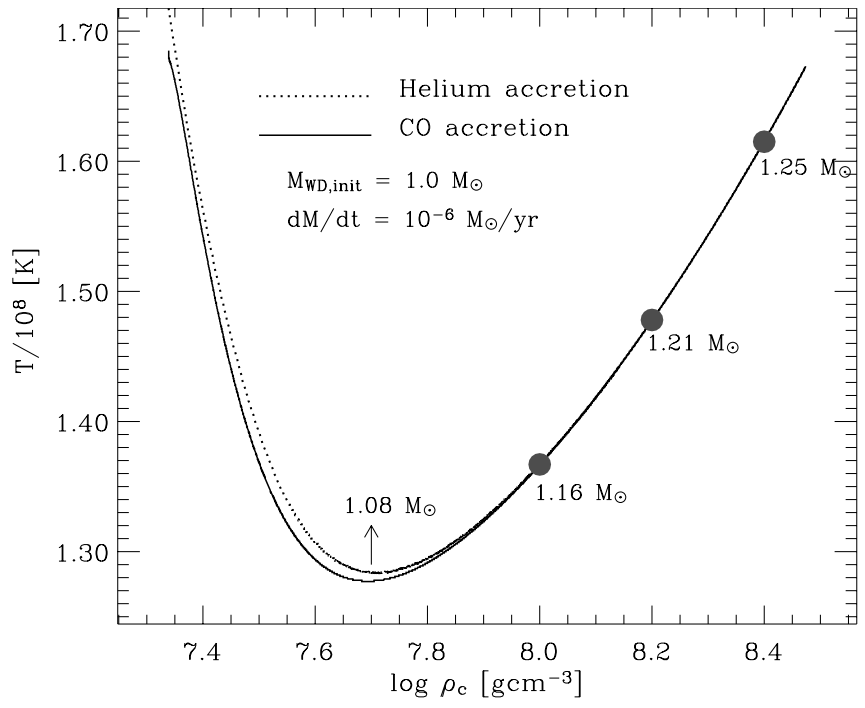

Fig. 3. Evolution of central density and temperature in non-rotating accreting $\mathrm{CO}$ white dwarfs. The initial mass is $1.0 M_{\odot}$ and the accretion rate is $10^{-6} M_{\odot} / y r$. The dotted line denotes the case of helium accretion, and the dashed line the case of carbon-oxygen accretion. The initial central temperature was $T_{\mathrm{c}, \text { init }}=2.29 \times 10^{8} \mathrm{~K}$ for the helium-accreting white dwarf and and $T_{\text {c,init }}=1.69 \times 10^{8} \mathrm{~K}$ for the CO-accreting one. The numbers next to the filled circles denote the white dwarf mass in solar masses.

enriched such that $X_{\mathrm{C}}=X_{\mathrm{O}}=0.487$. In this way, we assume that the accreted hydrogen or helium is immediately converted to carbon and oxygen by shell burning. This assumption, however, does not affect the advanced thermal evolution of the degenerate core of a white dwarf as demonstrated in Fig. 3. In this figure, the evolution of the central density and temperature of a non-rotating white dwarf that accretes matter at a rate of with $\dot{M}=10^{-6} M_{\odot} / \mathrm{yr}$ is shown for two cases. One case assumes $\mathrm{CO}$ accretion, the other case assumes helium accretion. In the latter case, we have followed the helium shell burning and thereby induced thermal pulses up to $M_{\mathrm{WD}}=1.2 M_{\odot}$. Although a slight difference appears when $M_{\mathrm{WD}} \lesssim 1.1 M_{\odot}$, which is due to the different initial temperatures as indicated in the figure caption, the two sequences converge when $M_{\mathrm{WD}} \gtrsim$ $1.1 M_{\odot}$ due to the dominance of the compressional heating over the thermal structure. Therefore, we can assume safely that the thermal evolution of the white dwarf interior does not change significantly even if we neglect the shell burning, as long as rapid accretion is ensured.

Since non-magnetic white dwarfs in close binary systems are thought to accrete matter through a Keplerian disk, the accreted matter may carry angular momentum of about the local Keplerian value at the white dwarf equator (cf. Durisen 1977; Ritter 1985). Langer et al. (2000) pointed out that a white dwarf will reach overcritical rotation well before growing to the Chandrasekhar limit if it gains angular momentum with the Keplerian value, assuming rigid body rotation (see also Livio \& Pringle 1998; Uenishi et al. 2003). Our preliminary calculations show that overcritical rotation may be reached even earlier at the white dwarf surface, as the angular momentum transport time scale is finite (Yoon \& Langer 2002). Interestingly, Paczyński (1991) and Popham \& Narayan (1991) found a possibility for the angular momentum to be transported from the white dwarf into the disk by viscous effects when the white dwarf rotates at near break-up velocity, without preventing the continued efficient mass accretion. Based on this picture, we limit the angular momentum gain such that no angular momentum is allowed to be transferred onto an accreting white dwarf when its surface rotates at the Keplerian value at its equator:

$j_{\mathrm{acc}}= \begin{cases}f \cdot j_{\mathrm{K}} & \text { if } v_{\mathrm{s}}<v_{\mathrm{K}} \\ 0 & \text { if } v_{\mathrm{s}}=v_{\mathrm{K}}\end{cases}$

where $j_{\text {acc }}$ denotes the specific angular momentum carried by the accreted matter, $v_{\mathrm{s}}$ the surface velocity of the accreting white dwarf at its equator, $v_{\mathrm{K}}$ and $j_{\mathrm{K}}$ the Keplerian value of the rotation velocity and the specific angular momentum at the white dwarf equator, respectively: $v_{\mathrm{K}}=\left(G M_{\mathrm{WD}} / R_{\mathrm{WD}}\right)^{1 / 2}$ and $j_{\mathrm{K}}=v_{\mathrm{K}} R_{\mathrm{WD}}$. The dimensionless parameter $f$ determines which fraction of the Keplerian value is actually accreted in a white dwarf when $v_{\mathrm{s}}<v_{\mathrm{K}}$.

As mentioned earlier, we do not consider nuclear shell burning in our simulations. Although this may not change the thermal history in the white dwarf core, the surface conditions can be affected by this simplification: the white dwarf envelope may be hotter and more extended with shell burning, which may lead to a stronger Eddington-Sweet circulation and different values for $j_{\mathrm{K}}$. To investigate how the results are affected by this uncertainty, we consider three different values for $f: 0.3$, 0.5 and 1.0 .

In calibrating the chemical mixing efficiencies in massive stars, Heger et al. (2000) introduced the factor $f_{\mu}$ such that $\nabla_{\mu}$ is replaced by $f_{\mu} \nabla_{\mu}$, with $f_{\mu}=0.05$ giving the best reproduction of the observed enhancement of nitrogen at the stellar surface in the mass range $10 M_{\odot}$ to $20 M_{\odot}$. Although this result is deduced from massive stars and might not apply to white dwarfs, we keep the value of $f_{\mu}=0.05$ for most of our models. However, to check the sensitivity of our results to this parameter, models with $f_{\mu}=1.0$ are also calculated (Sect. 4.3.4).

Table 2 gives an overview of the computed model sequences. The first column gives the model sequence designation, where the letters $\mathrm{A}, \mathrm{B}$ and $\mathrm{C}$ denote the cases with $f=$ $1.0,0.5$ and 0.3 respectively, all for a fixed $f_{\mu}$ of 0.05 . Model sequences with index D have $f=1.0$ and $f_{\mu}=1.0$. The index $\mathrm{T}$ indicates test sequences where the effects of rotational energy dissipation (Eq. (16)) are neglected.

\section{Results}

In this section, we present the results of our simulations, which are summarized in Tables 2 and 3. In these tables, key properties of the white dwarf models are given for three different epochs, i.e., when the ratio of the rotational energy to the gravitational potential energy $E_{\text {rot }} /|W|$ reaches $0.10,0.14$ and 0.18 . The implications of these numbers are discussed in Sect. 5. In the following subsections, after discussing the thermal evolution of our models, we focus on the processes of the angular momentum transport in the interior of accreting white dwarfs and its consequences for the white dwarf structure. 
Table 2. Properties of the computed model sequences. The first column gives the system number. The other columns have the following meanings. $M_{\text {init }}$ : initial mass, $\dot{M}$ : accretion rate, $f$ : fraction of $j_{\mathrm{K}}$ of the accreted matter (see Eq. (18)), $f_{\mu}$ : efficiency parameter of the $\mu$ gradient, $\dot{E}_{\text {rot }}$ : rotational energy dissipation by friction, $M_{0.10}$ : white dwarf mass when the ratio of the rotational energy to the gravitational potential energy $(T / W)$ reaches 0.10 . The remaining physical quantities are also estimated at this point, i.e., when $T / W=0.10 . \rho_{\mathrm{c}, 0.10}$ and $T_{\mathrm{c}, 0.10}$ : central density and temperature. $R_{0.10}$ : radius of white dwarf defined on the sphere of the volume of the equipotential surface. $J_{0.10}$ : total angular momentum, $\Omega_{0.10}$ : moment-of-inertia-weighted mean of angular velocity.

\begin{tabular}{|c|c|c|c|c|c|c|c|c|c|c|c|}
\hline No. & $M_{\text {init }}$ & $\dot{M}$ & $f$ & $\overline{f_{\mu}}$ & $\dot{E}_{\text {rot }}$ & $M_{0.10}$ & $\rho_{\mathrm{c}, 0.10}$ & $T_{\mathrm{c}, 0.10}$ & $R_{0.10}$ & $J_{0.10}$ & $\Omega_{0.10}$ \\
\hline & & $10^{-7}$ & & & & & $10^{8}$ & $10^{8}$ & 0.01 & $10^{50}$ & \\
\hline & $M_{\odot}$ & $M_{\odot} / \mathrm{yr}$ & & & & $M_{\odot}$ & $\mathrm{g} / \mathrm{cm}^{3}$ & $\mathrm{~K}$ & $R_{\odot}$ & erg $\mathrm{s}$ & $\mathrm{rad} / \mathrm{s}$ \\
\hline A1 & 0.8 & 3.0 & 1.0 & 0.05 & Yes & 1.16 & 0.29 & 0.92 & 1.22 & 1.10 & 0.68 \\
\hline $\mathrm{A} 2$ & 0.8 & 5.0 & 1.0 & 0.05 & Yes & 1.18 & 0.32 & 0.97 & 1.26 & 1.13 & 0.70 \\
\hline $\mathrm{A} 3$ & 0.8 & 7.0 & 1.0 & 0.05 & Yes & 1.19 & 0.33 & 1.01 & 1.34 & 1.14 & 0.69 \\
\hline A4 & 0.8 & 10.0 & 1.0 & 0.05 & Yes & 1.21 & 0.35 & 1.07 & 1.39 & 1.17 & 0.71 \\
\hline A5 & 0.9 & 3.0 & 1.0 & 0.05 & Yes & 1.28 & 0.58 & 1.02 & 1.02 & 1.18 & 0.95 \\
\hline A6 & 0.9 & 5.0 & 1.0 & 0.05 & Yes & 1.30 & 0.64 & 1.11 & 1.03 & 1.21 & 0.99 \\
\hline A7 & 0.9 & 7.0 & 1.0 & 0.05 & Yes & 1.32 & 0.69 & 1.17 & 1.03 & 1.23 & 1.02 \\
\hline A8 & 0.9 & 10.0 & 1.0 & 0.05 & Yes & 1.34 & 0.75 & 1.23 & 1.05 & 1.25 & 1.06 \\
\hline A9 & 1.0 & 3.0 & 1.0 & 0.05 & Yes & 1.41 & 1.30 & 1.14 & 0.82 & 1.26 & 1.40 \\
\hline A10 & 1.0 & 5.0 & 1.0 & 0.05 & Yes & 1.42 & 1.39 & 1.22 & 0.83 & 1.25 & 1.43 \\
\hline A11 & 1.0 & 7.0 & 1.0 & 0.05 & Yes & 1.43 & 1.52 & 1.28 & 0.82 & 1.27 & 1.49 \\
\hline A 12 & 1.0 & 10.0 & 1.0 & 0.05 & Yes & 1.45 & 1.67 & 1.36 & 0.83 & 1.28 & 1.56 \\
\hline B1 & 0.8 & 3.0 & 0.5 & 0.05 & Yes & 1.21 & 0.36 & 0.96 & 1.17 & 1.17 & 0.76 \\
\hline B2 & 0.8 & 5.0 & 0.5 & 0.05 & Yes & 1.25 & 0.42 & 1.04 & 1.20 & 1.22 & 0.81 \\
\hline B3 & 0.8 & 7.0 & 0.5 & 0.05 & Yes & 1.26 & 0.44 & 1.09 & 1.24 & 1.23 & 0.82 \\
\hline B4 & 0.8 & 10.0 & 0.5 & 0.05 & Yes & 1.30 & 0.52 & 1.16 & 1.23 & 1.27 & 0.89 \\
\hline B5 & 0.9 & 3.0 & 0.5 & 0.05 & Yes & 1.32 & 0.69 & 1.03 & 0.97 & 1.24 & 1.04 \\
\hline B6 & 0.9 & 5.0 & 0.5 & 0.05 & Yes & 1.35 & 0.79 & 1.12 & 0.97 & 1.27 & 1.11 \\
\hline B7 & 0.9 & 7.0 & 0.5 & 0.05 & Yes & 1.38 & 0.90 & 1.12 & 0.96 & 1.29 & 1.18 \\
\hline B8 & 0.9 & 10.0 & 0.5 & 0.05 & Yes & 1.41 & 1.04 & 1.27 & 0.97 & 1.32 & 1.26 \\
\hline B9 & 1.0 & 3.0 & 0.5 & 0.05 & Yes & 1.45 & 1.61 & 1.18 & 0.78 & 1.30 & 1.56 \\
\hline B10 & 1.0 & 5.0 & 0.5 & 0.05 & Yes & 1.46 & 1.76 & 1.27 & 0.78 & 1.30 & 1.62 \\
\hline B11 & 1.0 & 7.0 & 0.5 & 0.05 & Yes & 1.48 & 2.00 & 1.34 & 0.78 & 1.31 & 1.72 \\
\hline B12 & 1.0 & 10.0 & 0.5 & 0.05 & Yes & 1.51 & 2.33 & 1.44 & 0.77 & 1.32 & 1.84 \\
\hline $\mathrm{C} 1$ & 0.8 & 3.0 & 0.3 & 0.05 & Yes & 1.53 & 1.55 & 1.17 & 0.77 & 1.48 & 1.57 \\
\hline $\mathrm{C} 2$ & 0.8 & 5.0 & 0.3 & 0.05 & Yes & 1.50 & 1.25 & 1.19 & 0.84 & 1.46 & 1.41 \\
\hline $\mathrm{C} 3$ & 0.8 & 7.0 & 0.3 & 0.05 & Yes & 1.46 & 1.05 & 1.20 & 0.90 & 1.44 & 1.29 \\
\hline $\mathrm{C} 4$ & 0.8 & 10.0 & 0.3 & 0.05 & Yes & 1.42 & 0.85 & 1.21 & 1.01 & 1.40 & 1.15 \\
\hline C9 & 1.0 & 3.0 & 0.3 & 0.05 & Yes & 1.73 & 19.0 & 2.16 & 0.42 & 1.25 & 5.03 \\
\hline $\mathrm{C} 10$ & 1.0 & 5.0 & 0.3 & 0.05 & Yes & 1.72 & 16.2 & 2.04 & 0.44 & 1.27 & 4.65 \\
\hline C11 & 1.0 & 7.0 & 0.3 & 0.05 & Yes & 1.72 & 14.3 & 2.06 & 0.46 & 1.29 & 4.38 \\
\hline $\mathrm{C} 12$ & 1.0 & 7.0 & 0.3 & 0.05 & Yes & 1.71 & 12.1 & 2.01 & 0.49 & 1.31 & 4.04 \\
\hline D2 & 0.8 & 5.0 & 1.0 & 1.00 & Yes & 1.18 & 0.32 & 0.97 & 1.27 & 1.13 & 0.69 \\
\hline D6 & 0.9 & 5.0 & 1.0 & 1.00 & Yes & 1.30 & 0.64 & 1.11 & 1.03 & 1.21 & 0.99 \\
\hline $\mathrm{T} 2$ & 0.8 & 5.0 & 1.0 & 0.05 & No & 1.12 & 0.27 & 0.95 & 1.24 & 1.02 & 0.63 \\
\hline T6 & 0.9 & 5.0 & 1.0 & 0.05 & No & 1.26 & 0.56 & 1.03 & 0.97 & 1.15 & 0.93 \\
\hline $\mathrm{T} 10$ & 1.0 & 5.0 & 1.0 & 0.05 & No & 1.40 & 1.24 & 1.14 & 0.81 & 1.24 & 1.37 \\
\hline
\end{tabular}

\subsection{Thermal evolution}

The thermal evolution of non-rotating accreting white dwarfs has been studied by many authors (e.g. Iben 1982; Nomoto 1982; Sion 1995; Townsley \& Bildsten 2002). Piersanti et al. (2003) compared rigidly rotating white dwarf models with nonrotating ones for $\mathrm{CO}$-accretion at various accretion rates. They found that a rotating white dwarf is generally cooler than its non-rotating counterpart, which is a natural consequence of the lifting effect of the centrifugal force. This conclusion holds also in our differentially rotating white dwarf models (Fig. 4). However, as we shall see in Sect. 4.2, the thermal structure in an accreting white dwarf is found to have an interesting consequence for the redistribution of angular momentum, as it 
Table 3. Continued from Table 2. The symbols in the columns have the same meaning with those in Table 3 , but when $T / W=0.14$ and $T / W=0.18$ for the indices 0.14 and 0.18 , respectively.

\begin{tabular}{|c|c|c|c|c|c|c|c|c|c|c|c|c|}
\hline No. & $M_{0.14}$ & $\rho_{\mathrm{c}, 0.14}$ & $T_{\mathrm{c}, 0.14}$ & $R_{0.14}$ & $J_{0.14}$ & $\Omega_{0.14}$ & $M_{0.18}$ & $\rho_{\mathrm{c}, 0.18}$ & $T_{\mathrm{c}, 0.18}$ & $R_{0.18}$ & $J_{0.18}$ & $\Omega_{0.18}$ \\
\hline & & $10^{8}$ & $10^{8}$ & 0.01 & $10^{50}$ & & & $10^{8}$ & $10^{8}$ & 0.01 & $10^{50}$ & \\
\hline & $M_{\odot}$ & $\mathrm{g} / \mathrm{cm}^{3}$ & K & $R_{\odot}$ & erg $\mathrm{s}$ & $\mathrm{rad} / \mathrm{s}$ & $M_{\odot}$ & $\mathrm{g} / \mathrm{cm}^{3}$ & K & $R_{\odot}$ & erg s & $\mathrm{rad} / \mathrm{s}$ \\
\hline A1 & 1.31 & 0.42 & 0.94 & 1.10 & 1.54 & 0.97 & 1.45 & 0.56 & 0.96 & 1.01 & 1.97 & 1.26 \\
\hline A2 & 1.34 & 0.47 & 1.01 & 1.11 & 1.58 & 1.02 & 1.47 & 0.63 & 1.04 & 1.01 & 1.99 & 1.32 \\
\hline A3 & 1.35 & 0.49 & 1.06 & 1.13 & 1.59 & 1.03 & 1.48 & 0.66 & 1.09 & 1.03 & 2.00 & 1.34 \\
\hline A4 & 1.37 & 0.54 & 1.12 & 1.15 & 1.63 & 1.07 & 1.50 & 0.73 & 1.15 & 1.04 & 2.03 & 1.40 \\
\hline A5 & 1.42 & 0.78 & 1.03 & 0.93 & 1.62 & 1.30 & 1.55 & 1.04 & 1.74 & 0.87 & 2.02 & 1.68 \\
\hline A6 & 1.44 & 0.87 & 1.11 & 0.93 & 1.63 & 1.36 & 1.56 & 1.15 & 1.15 & 0.87 & 2.01 & 1.74 \\
\hline A7 & 1.46 & 0.97 & 1.17 & 0.93 & 1.65 & 1.42 & 1.58 & 1.28 & 1.21 & 0.85 & 2.03 & 1.83 \\
\hline A8 & 1.48 & 1.07 & 1.23 & 0.93 & 1.67 & 1.48 & 1.60 & 1.42 & 1.28 & 0.85 & 2.04 & 1.92 \\
\hline A9 & 1.55 & 1.78 & 1.18 & 0.76 & 1.66 & 1.90 & 1.69 & 2.56 & 1.28 & 0.69 & 2.03 & 2.54 \\
\hline A10 & 1.55 & 1.88 & 1.26 & 0.77 & 1.65 & 1.94 & 1.67 & 2.55 & 1.33 & 0.71 & 2.01 & 2.52 \\
\hline A11 & 1.57 & 2.08 & 1.33 & 0.76 & 1.66 & 2.03 & 1.69 & 2.82 & 1.40 & 0.70 & 2.00 & 2.63 \\
\hline A 12 & 1.58 & 2.31 & 1.40 & 0.75 & 1.66 & 2.12 & 1.70 & 3.13 & 1.49 & 0.70 & 2.00 & 2.75 \\
\hline B1 & 1.36 & 0.51 & 0.96 & 1.06 & 1.61 & 1.07 & 1.50 & 0.71 & 1.00 & 0.97 & 2.03 & 1.41 \\
\hline B2 & 1.40 & 0.62 & 1.05 & 1.05 & 1.66 & 1.16 & 1.54 & 0.85 & 1.09 & 0.96 & 2.07 & 1.53 \\
\hline B3 & 1.41 & 0.65 & 1.10 & 1.05 & 1.67 & 1.19 & 1.55 & 0.89 & 1.14 & 0.96 & 2.07 & 1.55 \\
\hline B4 & 1.45 & 0.78 & 1.17 & 1.06 & 1.71 & 1.28 & 1.58 & 1.07 & 1.22 & 0.95 & 2.09 & 1.69 \\
\hline B5 & 1.46 & 0.95 & 1.06 & 0.90 & 1.66 & 1.43 & 1.60 & 1.32 & 1.12 & 0.82 & 2.06 & 1.88 \\
\hline B6 & 1.49 & 1.12 & 1.15 & 0.89 & 1.68 & 1.54 & 1.63 & 1.56 & 1.22 & 0.81 & 2.07 & 2.02 \\
\hline B7 & 1.52 & 1.28 & 1.22 & 0.86 & 1.70 & 1.63 & 1.65 & 1.79 & 1.29 & 0.81 & 2.07 & 2.15 \\
\hline B8 & 1.55 & 1.52 & 1.31 & 0.87 & 1.72 & 1.76 & 1.67 & 2.12 & 1.39 & 0.77 & 2.07 & 2.32 \\
\hline B9 & 1.60 & 2.38 & 1.36 & 0.71 & 1.70 & 2.19 & 1.75 & 3.94 & 1.40 & 0.62 & 2.05 & 3.13 \\
\hline B10 & 1.60 & 2.52 & 1.34 & 0.72 & 1.68 & 2.23 & 1.74 & 3.81 & 1.46 & 0.64 & 2.02 & 3.05 \\
\hline B11 & 1.62 & 2.90 & 1.42 & 0.70 & 1.68 & 2.38 & 1.75 & 4.32 & 1.54 & 0.64 & 2.01 & 3.22 \\
\hline B12 & 1.64 & 3.43 & 1.53 & 0.68 & 1.68 & 2.56 & 1.75 & 4.91 & 1.65 & 0.61 & 1.98 & 3.42 \\
\hline $\mathrm{C} 1$ & 1.74 & 3.36 & 1.36 & 0.64 & 1.87 & 2.62 & 1.87 & 6.30 & 1.57 & 0.54 & 2.13 & 3.99 \\
\hline $\mathrm{C} 2$ & 1.71 & 2.66 & 1.38 & 0.69 & 1.89 & 2.35 & 1.86 & 5.02 & 1.58 & 0.59 & 2.17 & 3.58 \\
\hline C3 & 1.68 & 2.18 & 1.37 & 0.74 & 1.89 & 2.13 & 1.84 & 4.13 & 1.57 & 0.63 & 2.20 & 3.27 \\
\hline $\mathrm{C} 4$ & 1.64 & 1.69 & 1.35 & 0.81 & 1.88 & 1.88 & 1.81 & 3.20 & 1.54 & 0.69 & 2.23 & 2.89 \\
\hline D2 & 1.34 & 0.47 & 1.01 & 1.11 & 1.58 & 1.02 & 1.47 & 0.63 & 1.04 & 1.01 & 1.99 & 1.32 \\
\hline D6 & 1.44 & 0.87 & 1.11 & 0.94 & 1.63 & 1.36 & 1.56 & 1.15 & 1.15 & 0.87 & 2.01 & 1.74 \\
\hline $\mathrm{T} 2$ & 1.26 & 0.37 & 0.95 & 1.12 & 1.45 & 0.90 & 1.38 & 0.47 & 0.95 & 1.05 & 1.85 & 1.14 \\
\hline T6 & 1.39 & 0.72 & 1.02 & 0.94 & 1.57 & 1.25 & 1.51 & 0.72 & 1.02 & 0.94 & 1.57 & 1.25 \\
\hline $\mathrm{T} 10$ & 1.52 & 1.57 & 1.15 & 0.76 & 1.63 & 1.79 & 1.64 & 2.06 & 1.21 & 0.71 & 2.00 & 2.29 \\
\hline
\end{tabular}

affects the stability criterion for the dynamical shear instability. Therefore, before discussing the angular momentum transport, we examine the evolution of the thermal structure in our white dwarf models.

Figure 4 displays the temperature profiles of selected white dwarf models of different sequences. The non-rotating models (Fig. 4a) show a rapid increase of the central temperature, from $10^{8} \mathrm{~K}$ when $M_{\mathrm{WD}}=0.96 M_{\odot}$ to $2.7 \times 10^{8} \mathrm{~K}$ when $M_{\mathrm{WD}}=1.37 M_{\odot}$. In the corresponding rotating models (Figs. $4 \mathrm{~b}$ and c), the change in the central temperature is not significant in the considered period.
A comparison of Figs. $4 \mathrm{~b}$ and $4 \mathrm{c}$ shows the effects of rotational energy dissipation $\left(\dot{E}_{\text {rot }}\right)$ described by Eq. (16). In model sequence T6 (Fig. $4 \mathrm{~b}$ ), where $\dot{E}_{\text {rot }}$ is neglected, the white dwarf models are cooler than in the corresponding models with $\dot{E}_{\text {rot }}$ considered (sequence A6, Fig. 4c). As shown in Fig. 5, the rotational energy dissipation rate can be as high as $10^{3} \ldots 10^{4} \mathrm{erg} / \mathrm{g} / \mathrm{s}$ in the degenerate core, and even higher close to the surface. The integrated energy dissipation rate $\dot{E}_{\text {rot }}=\int \epsilon_{\text {diss }} \mathrm{d} M_{\mathrm{r}}$ reaches several times $10^{2} L_{\odot}$ in these models. This results in a heating of the region with a strong shear motion (cf. Fig. 6 below), with the consequence that the 

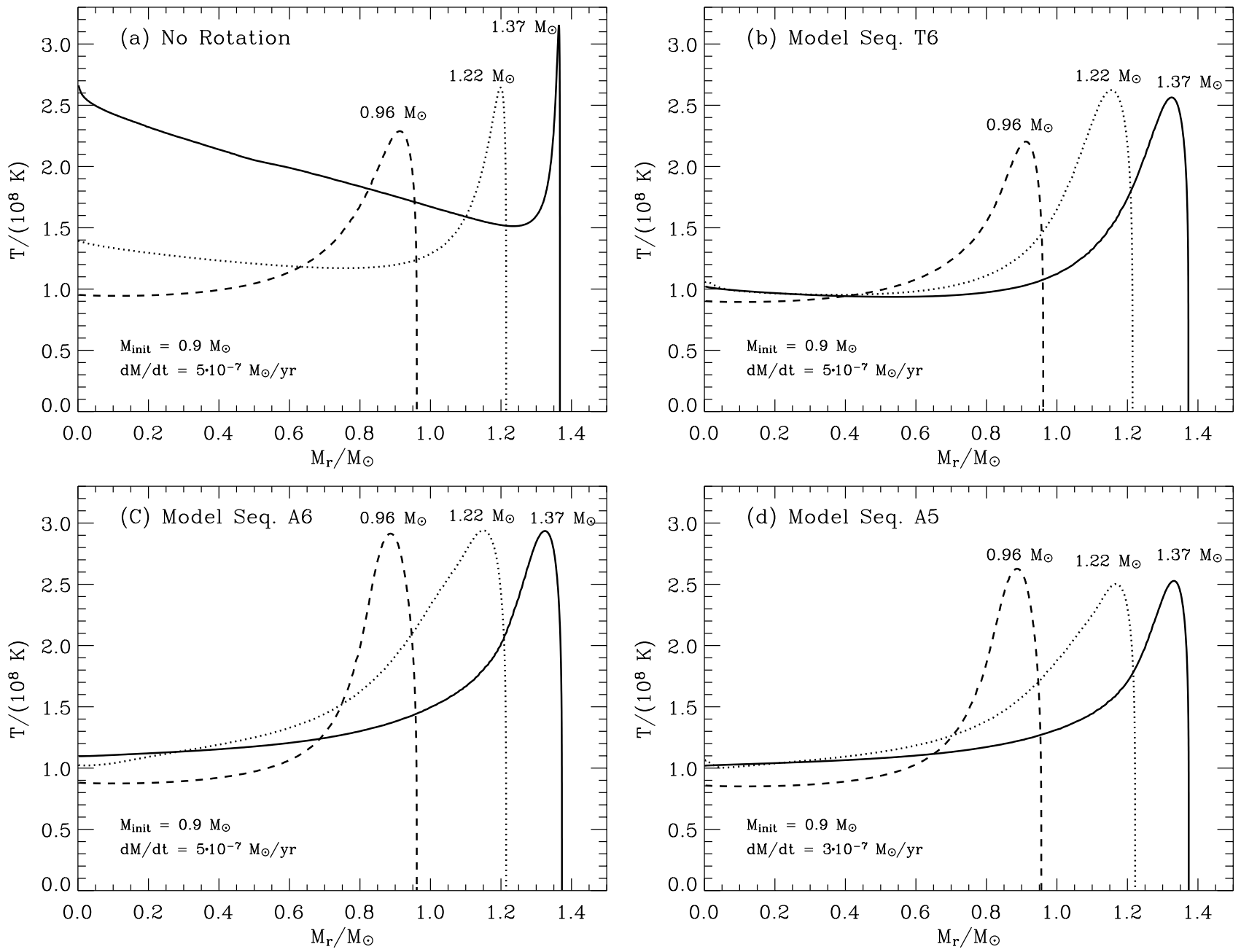

Fig. 4. Temperature as a function of the mass coordinate in white dwarf models when $M_{\mathrm{WD}}=0.96,1.22$ and $1.37 M_{\odot}$. a) non-rotating case with $M_{\text {init }}=0.9 M_{\odot}$ and $\left.\dot{M}=5 \times 10^{-7} ; \mathbf{b}\right)$ model sequence T6; c) model sequence A6; d) model sequence A6.

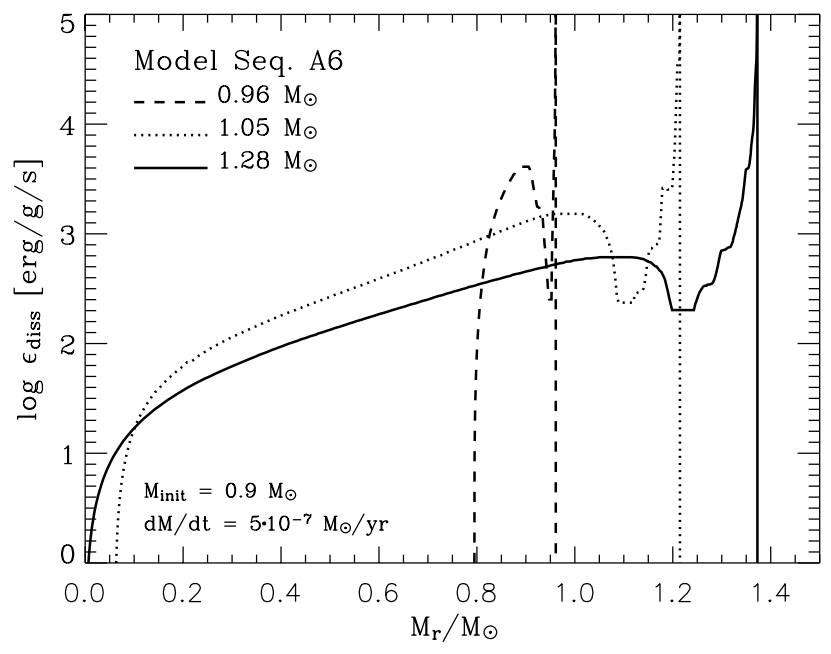

Fig. 5. Rotational energy dissipation rate (see Eq. (16)) as function of the mass coordinate, when $M_{\mathrm{WD}}=0.98,1.22$ and $1.37 M_{\odot}$ in model sequence A6.

temperature maxima when $M_{\mathrm{WD}}=0.96,1.22$ and $1.37 M_{\odot}$ in models of sequence A6 are higher than in the models of sequence T6. The accretion-induced heating is a sensitive function of the accretion rate: comparison of Fig. 4c with Fig. $4 d$ indicates that the white dwarf becomes significantly hotter with a higher accretion rate. The effects of the accretion rate and of $\dot{E}_{\text {rot }}$ for the angular momentum transport are discussed in Sect. 4.3.3.

Note that in all white dwarf models there exists an absolute temperature maximum in the outer layers of the degenerate core. Below this temperature peak the temperature gradient $\nabla$ becomes negative and large. This produces a strong buoyancy force in this region, since the Brunt-Väisälä frequency is proportional to $\nabla_{\mathrm{ad}}-\nabla$. The temperature peak moves outward as the white dwarf accretes more mass. This leads to changes in the local thermodynamic condition which plays a key role for the angular momentum transport from the outer envelope into the inner core, as shown in the next section.

\subsection{White dwarf spin and angular momentum transport}

Our white dwarf models are spun up as they gain angular momentum from the accreted matter, as described in Sect. 3.3. 

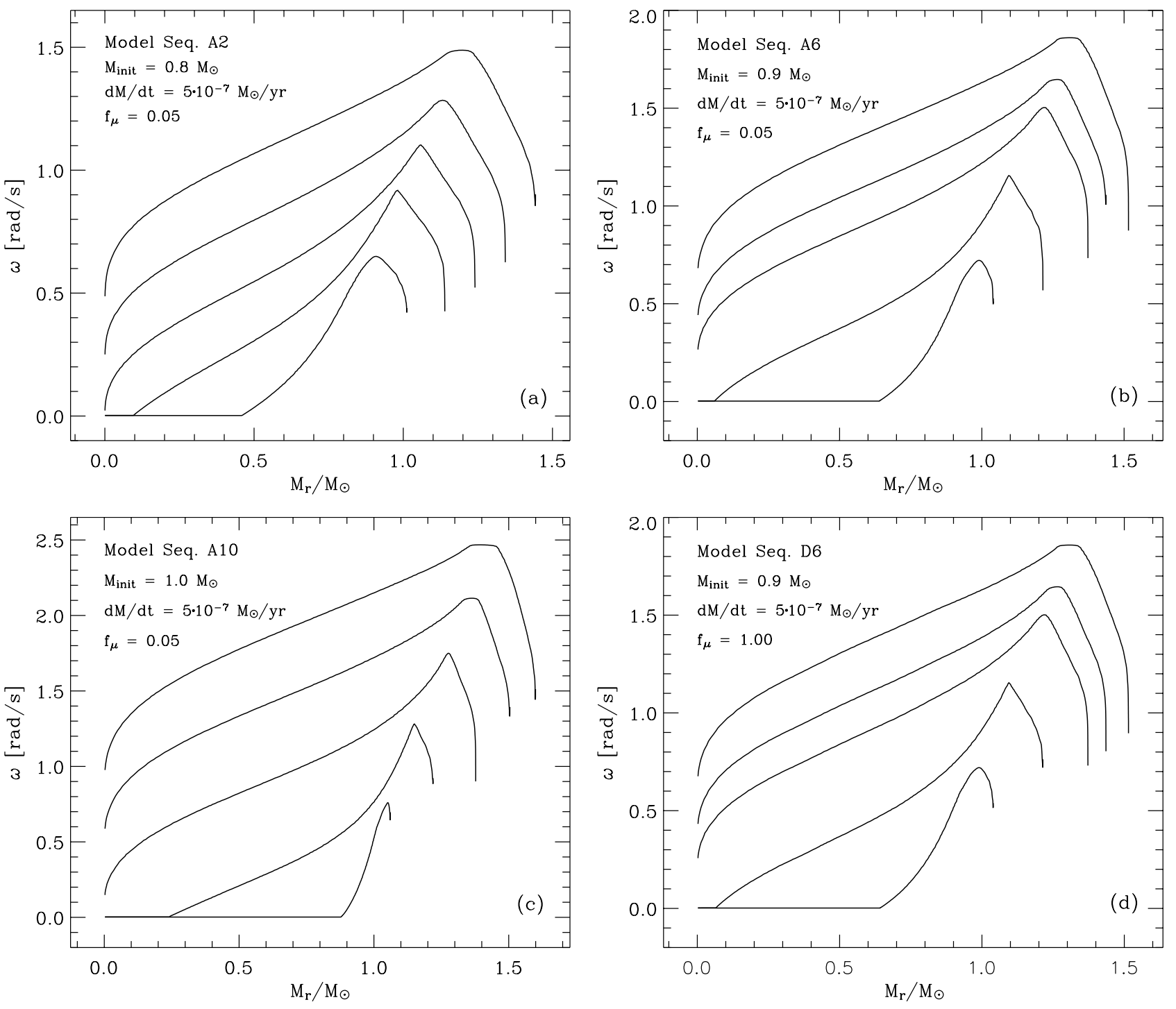

Fig. 6. Angular velocity as a function of the mass coordinate at different white dwarf masses. Panels a), b), c) and d) give results for model sequences A2, A6, A10 and D6, respectively (see Table 2).

Figure 6 shows the evolution of the angular velocity profiles in model sequences A2, A6, A10 and D6. Most strikingly, all white dwarf models rotate differentially, as predicted from the discussions in Sect. 2. Note also that in every white dwarf model in Fig. 6, a maximum angular velocity occurs, such that $\sigma>0$ in the inner core and $\sigma<0$ in the outer layers. This maximum comes into existence soon after the onset of mass accretion because the slowly rotating inner part contracts faster than the rapidly rotating surface layers as the total mass increases.

Since angular momentum is transported in the direction of decreasing angular velocity, this peak in $\omega$ serves as a bottleneck for the angular momentum transport from the outer envelope into the core. It prevents the outer envelope from slowing down efficiently by inward angular momentum transport. As a result, the surface continues to rotate close to the critical value throughout the evolution, severely limiting the angular momentum gain from the accreted matter. About $60 \%$ of the angular momentum of the accreted matter is rejected by the condition posed in Eq. (18), and only about $40 \%$ is actually retained by the time the white dwarf mass reaches $1.4 M_{\odot}$, in all model sequences (see Fig. 11 below).

Figure 7 shows the shear factor $\sigma$ as a function of the mass coordinate at two different evolutionary epochs $\left(M_{\mathrm{WD}}=1.26\right.$ and $1.37 M_{\odot}$ ) in model sequence A6. The dashed line gives the threshold value of the shear factor $\left(\sigma_{\text {DSI,crit }}\right)$. Note that the two, i.e., $\sigma$ and $\sigma_{\text {DSI,crit }}$ converge remarkably well in the white dwarf interior, i.e., in $M_{\mathrm{r}} \lesssim 1.1 M_{\odot}$ when $M_{\mathrm{WD}}=1.26 M_{\odot}$ and $M_{\mathrm{r}} \lesssim$ $1.2 M_{\odot}$ when $M_{\mathrm{WD}}=1.37 M_{\odot}$. This confirms the conclusion given in Sect. 2 that the degenerate core of an accreting white dwarf will rotate differentially with the shear strength near the threshold value for the dynamical instability. As already discussed in Sect. 2.2, this is because any strong shear motion with $\sigma>\sigma_{\text {DSI,crit }}$ cannot be retained for a long time and should decay to $\sigma_{\text {DSI,crit }}$ quickly via the dynamical shear instability, and because further angular momentum transport by other mechanisms requires a longer time scale compared to the accretion time scale. 


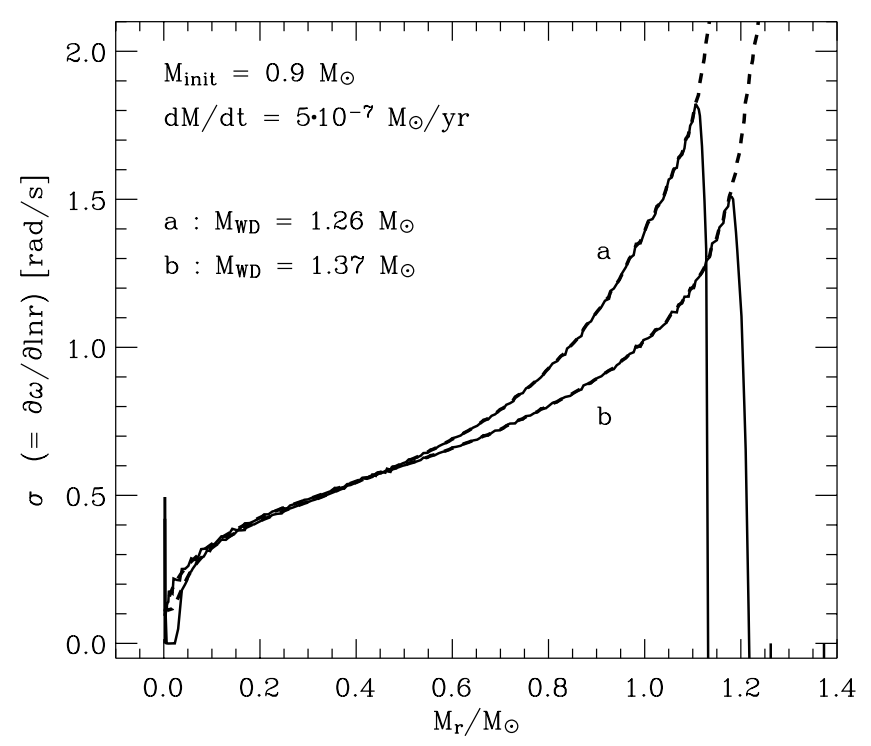

Fig. 7. Shear factor $\sigma(=\partial \omega / \partial \ln r)$ as a function of the mass coordinate in two different white dwarf models at $M_{\mathrm{WD}}=1.26 M_{\odot}$ and $1.37 M_{\odot}$ from model sequence A6. The dashed lines denote the threshold value of $\sigma$ for the dynamical shear instability (i.e., $\sigma_{\text {DSI,crit }}=\left(N^{2} / R_{\mathrm{i}, \mathrm{c}}\right)^{1 / 2}$; see Eq. (7)).

Figure 8 depicts the situation in more detail. The right hand panel of this figure shows the various diffusion coefficients as a function of the mass coordinate in the white dwarf models of sequence A6 when $M_{\mathrm{WD}}=1.26$ and $1.37 M_{\odot}$. This figure shows that the white dwarf consists of an inner dynamicalshear-unstable region and an outer region dominated by the secular shear instability and Eddington-Sweet circulations. Let us define $M_{\mathrm{r}, \mathrm{DSI}}$ as the mass coordinate at the point which divides the white dwarf into these two regions. $M_{\mathrm{r}, \mathrm{DSI}}$ changes with time not only due to the change of the shear strength, but also due to the evolution of the thermodynamic properties as explained below.

As shown in Sect. 4.1, the accretion-induced heating results in a temperature maximum in the outer region of an accreting white dwarf. A steep negative temperature gradient (i.e., $\nabla<0$ ) thus appears just below this temperature peak. The buoyancy force is enhanced in this region, which leads to stability against the dynamical shear instability (see Eqs. (3)-(6)). Therefore, the location of $M_{\mathrm{r}, \mathrm{DSI}}$ is below the region containing the strong temperature gradient, as demonstrated in Fig. 8.

According to Fig. 8, $M_{\mathrm{r} \text {,DSI }}$ moves outward as the white dwarf mass increases (see also Figs. 7 and 9). This outward shift of $M_{\mathrm{r}, \mathrm{DSI}}$ can be understood as follows. As shown in Fig. 7, the threshold value $\sigma_{\text {DSI,crit }}$ becomes smaller near $M_{\text {r,DSI }}$ as the white dwarf mass increases. Two processes contribute to this effect. First, the degeneracy at a given $M_{\mathrm{r}}$ becomes stronger as the white dwarf mass increases, reducing the buoyancy force. Second, the region with strong temperature stratification with $\nabla<0$ moves outward as indicated in Figs. 8a and $8 \mathrm{c}$. Consequently the dynamical-shear-unstable region becomes extended outward with time as shown in Figs. 8 and 9. The outward shift of $M_{\mathrm{r}, \mathrm{DSI}}$ allows the angular momentum from the outer layers to be transported inward, and thus leads to the outward shift of the position of the maximum angular velocity as shown in Fig. 9.

Figure 10 shows how much angular momentum is actually transported into the white dwarf interior in model sequence A6. Here $J\left(M_{\mathrm{r}}\right)=\int_{0}^{M_{\mathrm{r}}} j(m) \mathrm{d} m$ is the integrated angular momentum. In a rigidly rotating homogeneous body, we have $J\left(M_{\mathrm{r}}\right) / M_{r}^{5 / 3}=$ const., and therefore any deviation from the zero gradient in $J\left(M_{\mathrm{r}}\right) / M_{\mathrm{r}}^{5 / 3}$ may serve as a measure of the degree of differential rotation. A comparison of different evolutionary epochs demonstrates the flow of angular momentum through the mass shells, as $J\left(M_{\mathrm{r}}\right)$ and $J\left(M_{\mathrm{r}}\right) / M_{\mathrm{r}}^{5 / 3}$ would remain constant in a given mass shell throughout the evolution if no angular momentum were transported through this shell. Note that all the white dwarf models in this figure have a similar amount of angular momentum $\left(J \simeq 10^{50} \mathrm{erg} \mathrm{s}\right)$ at around $M_{\mathrm{r}} \sim 1.2 M_{\odot}$, which is close to the angular velocity peaks shown in Fig. 9. The angular momentum transport from this point into the further interior is shown to be efficient, due to the dynamical shear instability as described above.

We summarize the main conclusions from this section as follows. a) We find accreting white dwarf models to rotate differentially throughout their evolution; b) the dynamical shear instability is the most important mechanism for angular momentum transport in the highly degenerate core of our white dwarf models; c) the angular momentum gain from the accreted matter and the spin-up of our accreting white dwarfs is closely related to their restructuring and thermal evolution as their mass increases.

\subsection{Influence of physical assumptions}

Having understood the detailed processes of the angular momentum transport in an accreting white dwarf, let us consider the influence of the different initial conditions and physical assumptions on the evolution of accreting white dwarfs.

\subsubsection{Influence of the initial white dwarf mass}

Tables 2 and 3 give the white dwarf mass when the ratio of the rotational energy to the gravitational potential energy $\left(E_{\mathrm{rot}} /|W|\right)$ in each model sequence reaches $0.1,0.14$ and $0.18\left(M_{0.1}, M_{0.14}\right.$ and $\left.M_{0.18}\right)$. It is believed that a rapidly rotating white dwarf becomes secularly unstable when $E_{\text {rot }} /|W| \simeq 0.14$ (e.g. Durisen \& Imamura 1981). Imamura et al. (1995) found that this critical value can be lowered to about 0.1 for strong differential rotation (see detailed discussion in Sect. 5).

The tables show that the lower the initial mass, the higher the values of $M_{0.10}$ and $M_{0.14}$. For example, $M_{0.14}$ increases from $1.34 M_{\odot}$ to $1.55 M_{\odot}$ with a change of the initial mass from $0.8 M_{\odot}$ to $1.0 M_{\odot}$ in the case of $f=1$ (sequences A2 and A10). The total angular momentum also increases at a given $E_{\text {rot }} /|W|$ with higher initial mass. This tendency is simply due to the fact that more angular momentum gain is necessary to reach a certain amount of $E_{\text {rot }} /|W|$ if a white dwarf is more massive. 

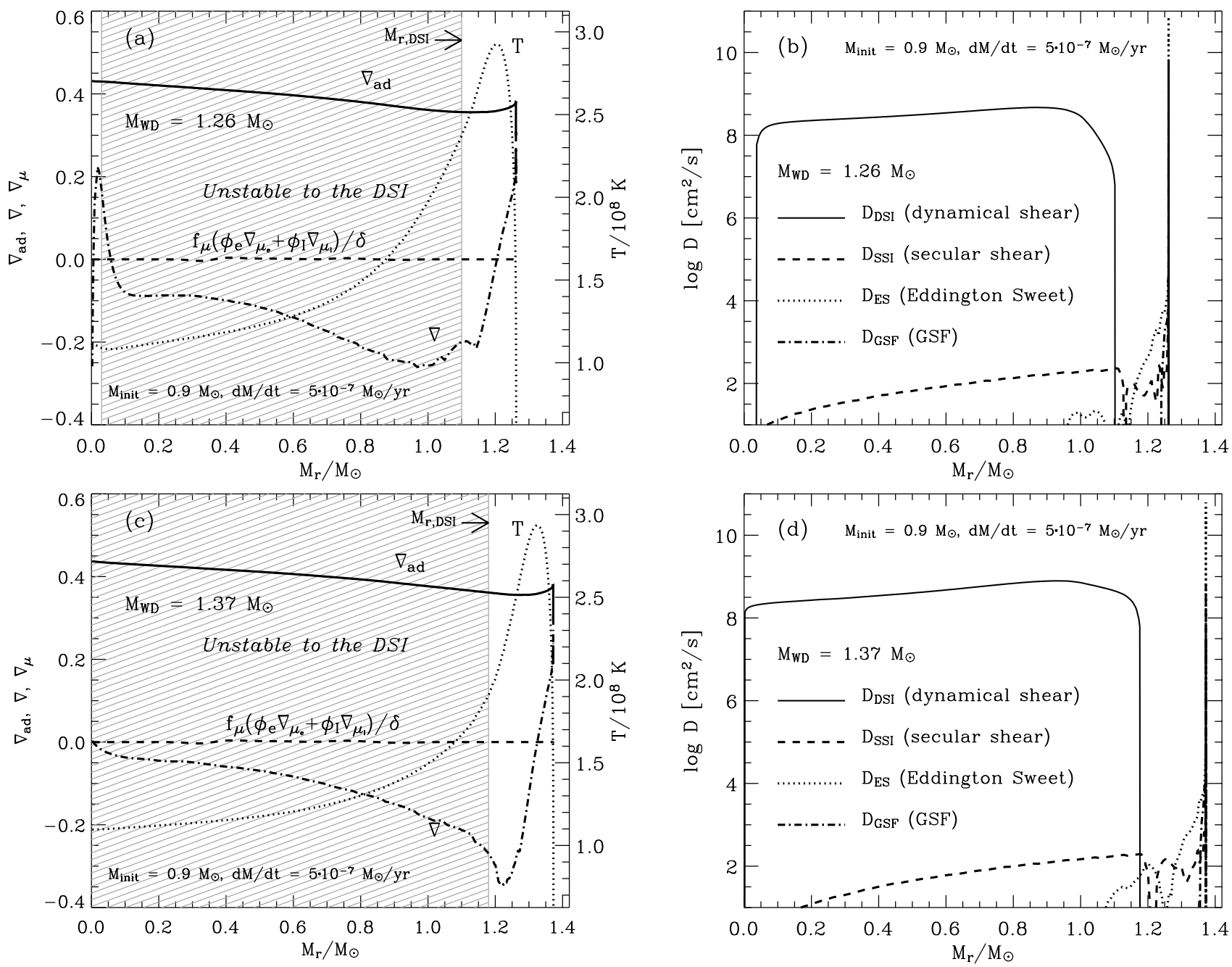

Fig. 8. a) Thermodynamic quantities as a function of the mass coordinate, in the white dwarf model of sequence A6 when $M_{\mathrm{WD}}=1.26 M_{\odot}$ : $\nabla_{\text {ad }}$ (solid line), $\nabla$ (dot-dashed line), $f_{\mu}\left(\varphi_{\mathrm{e}} \nabla_{\mu_{\mathrm{e}}}+\varphi_{\mathrm{I}} \nabla_{\mu_{\mathrm{I}}}\right) / \delta$ where $f_{\mu}=0.05$ (dashed line) and temperature (dotted line). The region where the white dwarf is unstable to the dynamical shear instability is hatched. b) Diffusion coefficients for rotationally induced hydrodynamic instabilities, as a function of the mass coordinate, in the white dwarf model of sequence A6 at $M_{\mathrm{WD}}=1.26 M_{\odot}$. The solid line denotes the diffusion coefficient for the dynamical shear instability, the dashed line, the dotted line and the dashed-dotted lines are for the secular shear instability, the Eddington-Sweet circulation, and the GSF instability respectively. c) Same as in a) but for $M_{\mathrm{WD}}=1.27 M_{\odot}$. d) Same as in b) but for $M_{\mathrm{WD}}=1.37 M_{\odot}$.

\subsubsection{Influence of the angular momentum gain parameter $f$}

As indicated in Tables 2 and 3, model sequences with $f=1.0$ and those with $f=0.5$ show some differences in the results. The adoption of $f=0.5$ yields a larger white dwarf mass at a given $E_{\text {rot }} /|W|$, by about $0.05-0.06 M_{\odot}$, which is a natural consequence of less angular momentum being accreted per unit time (Eq. (18)). This effect becomes more prominent with $f=0.3$, and $M_{0.1}$ increases by about $24 \%$ and $17 \%$ for $M_{\text {init }}=$ $0.8 M_{\odot}$ and $M_{\text {init }}=1.0 M_{\odot}$ respectively, compared to the case of $f=1$. Furthermore, the models with $M_{\text {init }}=1.0 M_{\odot}$ and $f=0.3$ reach central carbon ignition when $E_{\text {rot }} /|W|$ reaches about 0.11 .

Interestingly, the helium-accreting white dwarf models by Yoon et al. (2004), where $f=1$ is adopted, show similar $E_{\text {rot }} /|W|$ values at a given mass as the present CO-accreting white dwarf models with $f=0.3$. As mentioned in Sect. 3.2, this difference is mainly due to the fact that the angular momentum transport efficiency in the outermost layers is affected by the energy generation in the helium burning shell. In particular, the Eddington-Sweet circulation becomes more efficient due to shell burning, resulting in a more efficient outward angular momentum transport in the non-degenerate envelope (cf. Fig. 6). This causes a more severe restriction of the angular momentum gain from the accreted matter, due to the condition posed by Eq. (18). This implies that the history of the angular momentum gain may also be different for hydrogen-accreting cases.

However, these ambiguities concern only the actual amount of the angular momentum gain from the accreted matter, but do not affect the history of the angular momentum redistribution in the degenerate core, where differential rotation persists during the mass accretion phase (Sects. 2.2 and 4.2). In fact, despite 


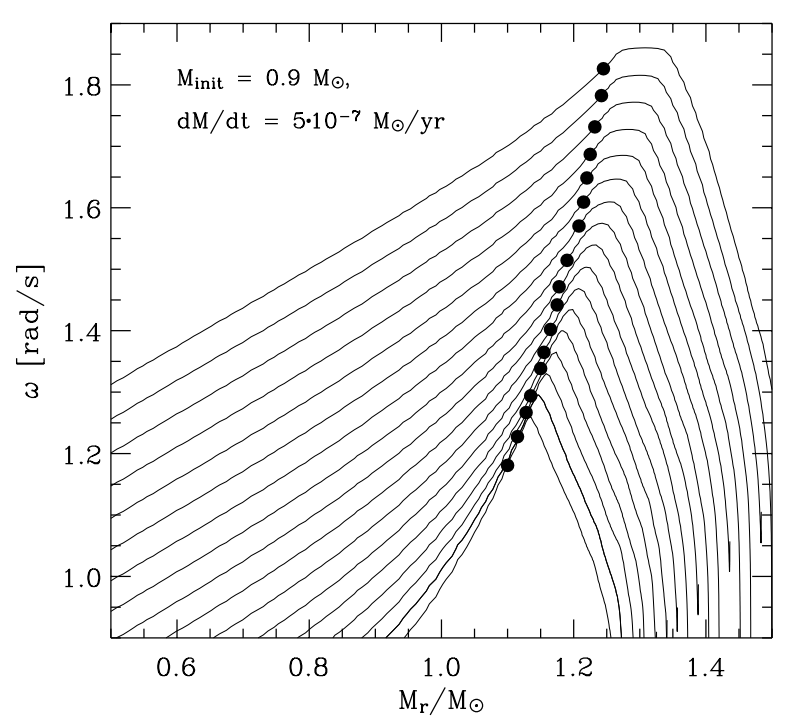

Fig. 9. Angular velocity profiles given as a function of the mass coordinate at 17 different evolutionary epochs of sequence A6: 1.26, 1.28, $1.30,1.31,1.33,1.34,1.36,1.37,1.38,1.40,1.42,1.44,1.45,1.47$, $1.48,1.50,1.52 M_{\odot}$, from the bottom to the top. The filled circles designate the mass coordinate $M_{\mathrm{rm}, \mathrm{DSI}}$ below which the white dwarf interior is dynamical shear unstable.

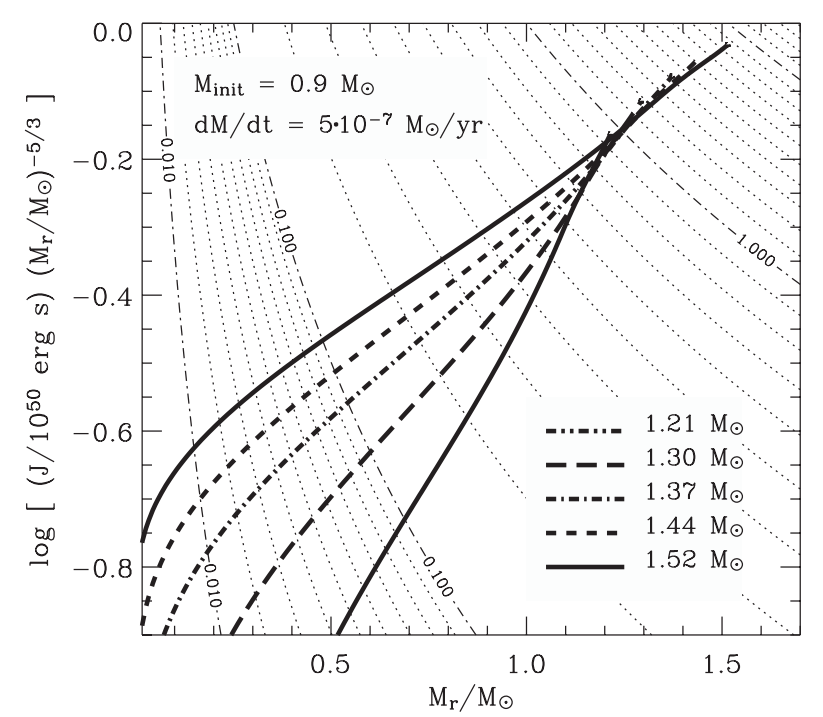

Fig. 10. Integrated angular momentum $J\left(M_{\mathrm{r}}\right)=\int_{0}^{M_{\mathrm{r}}} j(m) \mathrm{d} m$ divided by $M_{\mathrm{r}}^{5 / 3}$, as a function of the mass coordinate at five different evolutionary epochs of sequence A6: $M_{\mathrm{WD}}=1.21,1.30,1.37,1.44$ and $1.52 M_{\odot}$. The thin contour lines denote levels of constant $J$ in logarithmic scale, labeled with $\log \left(J / 10^{50} \mathrm{ergs}\right)$.

big differences in $f$, all model sequences show the same remarkable feature that carbon ignition is not reached even when $M_{\mathrm{WD}} \gtrsim 1.4 M_{\odot}$, due to differential rotation.

\subsubsection{Influence of accretion rate and dissipation}

Tables 2 and 3 show that with a given initial mass, the white dwarf mass at a given $E_{\text {rot }} /|W|$ increases for higher accretion rate. Figure 11 gives the accumulated angular momentum $\left(\Delta J_{\text {accreted }}\right)$ in the white dwarf, as well as the accumulated

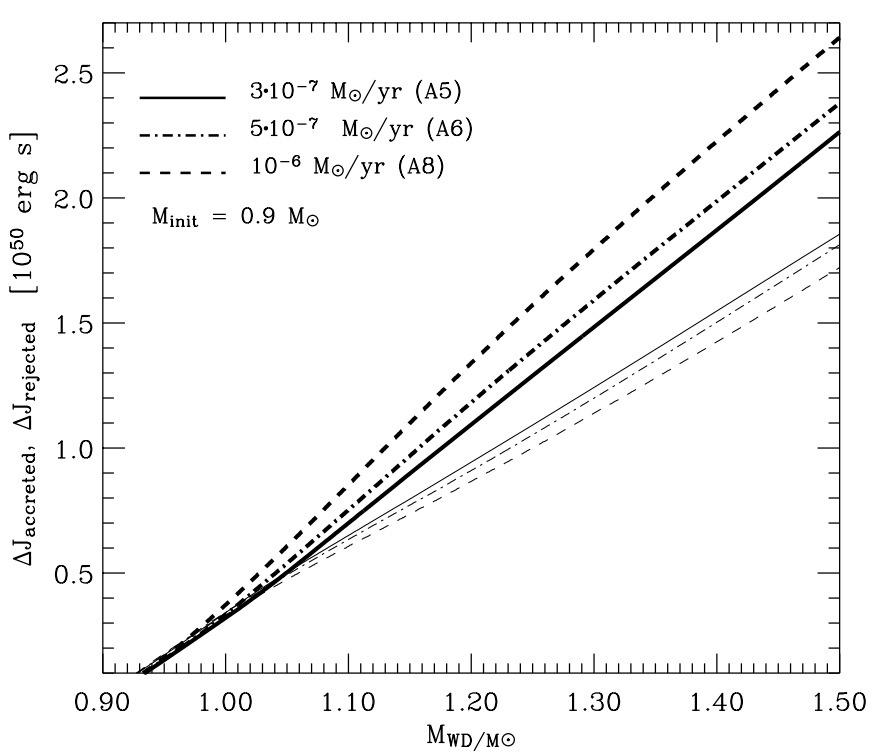

Fig. 11. The evolution of the accumulated angular momentum in model sequences A5, A6 and A10, given as a function of the white dwarf mass. The thin lines denote the accumulated total angular momentum in the white dwarf models. The thick lines give the accumulated rejected angular momentum by Eq. (18).

rejected angular momentum $\left(\Delta J_{\text {rejected }}\right)$ according to Eq. (18), as a function of the white dwarf mass, for sequences with $M_{\text {init }}=0.9 M_{\odot}$, and for three different accretion rates as indicated in the figure. It is shown that the higher the accretion rate, the more angular momentum is rejected and the less angular momentum is accreted, which is the reason for the higher white dwarf mass at a given $E_{\text {rot }} /|W|$ for a higher accretion rate.

This accretion rate dependence can be explained by two factors. First, with a lower accretion rate, a white dwarf gains a smaller amount of angular momentum per time and thus has more time to transport angular momentum into the white dwarf interior. Second, as already pointed out in Sect. 4.1, a higher accretion rate results in a stronger accretion-induced heating, which leads to higher temperatures inside the white dwarf (Fig. 4). This reduces the degeneracy in the white dwarf interior, and the buoyancy force becomes accordingly stronger. This thermal effect changes the stability condition for the dynamical shear instability in the white dwarf interior: the higher the accretion rate, the less susceptible to the dynamical shear instability it is, which in turn limits the angular momentum transfer from the outer envelope into the interior more severely.

We can also understand why the white dwarf mass at a given $E_{\text {rot }} /|W|$ is smaller when $\dot{E}_{\text {rot }}$ is neglected (Tables 2 and 3 ) in terms of different thermal structures: the white dwarf temperature becomes significantly lower without $\dot{E}_{\text {rot }}$, as shown in Fig. 4, resulting in a lower buoyancy force in the white dwarf interior and thus a more efficient angular momentum transport.

\subsubsection{Influence of chemical gradients}

In the model sequences discussed in the previous sections, the effect of $\mu$-gradients is significantly reduced by using $f_{\mu}=0.05$, as described in Sect. 3.3. In order to understand the 

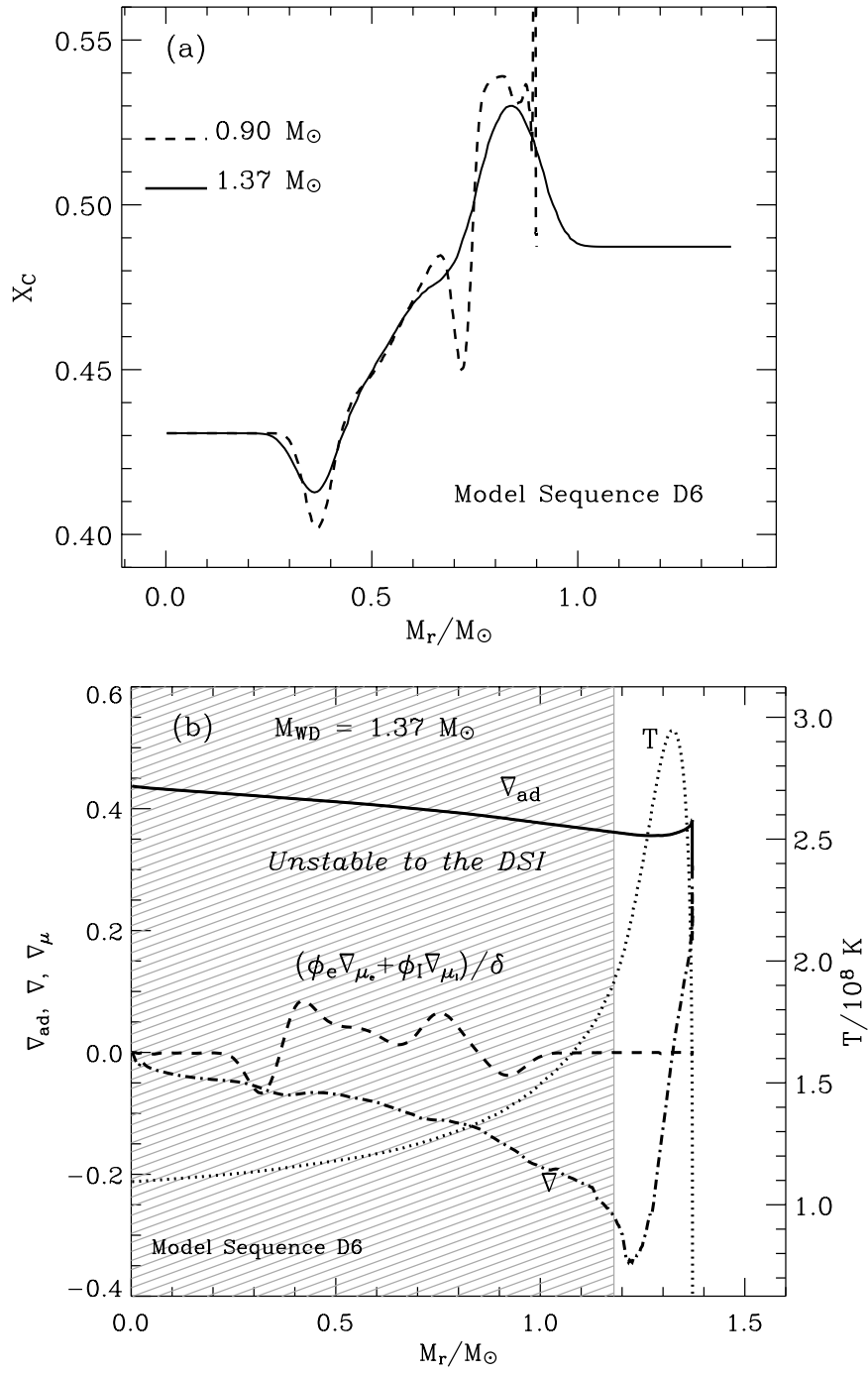

Fig. 12. a) Mass fraction of carbon as a function of the mass coordinate in two white dwarf models of sequence D6, at $M_{\mathrm{WD}}=0.9 M_{\odot}$ (initial model, dashed line) and $1.37 M_{\odot}$ (solid line). b) Thermodynamic quantities as a function of the mass coordinate for the same models: $\nabla_{\text {ad }}$ (solid line), $\nabla$ (dot-dashed line), $\left(\varphi_{\mathrm{e}} \nabla_{\mu_{\mathrm{e}}}+\varphi_{\mathrm{I}} \nabla_{\mu_{\mathrm{I}}}\right) / \delta$ (dashed line) and temperature (dotted line). The region where the white dwarf is unstable to the dynamical shear instability is hatched.

importance of chemical gradients for the angular momentum evolution, two model sequences (D2 and D6) are computed with $f_{\mu}=1.0$. The results are presented in Tables 2 and 3 . Interestingly, it is found that the adoption of different $f_{\mu}$ has hardly any effect. For instance, we have exactly the same values for $M_{0.1}, M_{0.14}$ and $M_{0.18}$ in the two corresponding model sequences A2 and D2, and also in A6 and D6.

Figure 12a shows the chemical structure in two white dwarf models of sequence D6, in the initial model $\left(0.9 M_{\odot}\right)$ and when $M_{\mathrm{WD}}=1.37 M_{\odot}$. The model at $1.37 M_{\odot}$ shows that although the rotationally induced chemical mixing smoothes out the chemical structure significantly, a strong stratification in the chemical composition persists in the range $M_{\mathrm{r}}=0.4-0.8 M_{\odot}$. Nevertheless, its contribution to the total buoyancy force turns out to be too small to suppress the dynamical shear

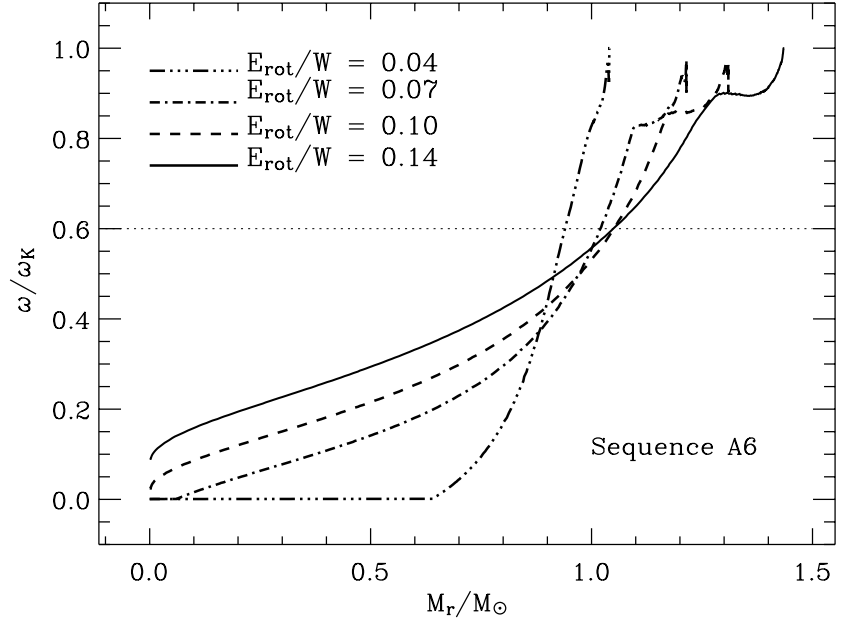

Fig. 13. Angular velocity normalized to the local Keplerian value, as a function of the mass coordinate in white dwarf models of sequence A6, when $E_{\text {rot }} /|W|=0.04,0.07,0.10$ and 0.14 .

instability, as implied in Fig. 12b. The term $\left(\varphi_{\mathrm{e}} \nabla_{\mu_{\mathrm{e}}}+\varphi_{\mathrm{I}} \nabla_{\mu_{\mathrm{I}}}\right) / \delta$ has a maximum value of about 0.1 , which is only $25 \%$ of $\nabla_{\mathrm{ad}}$.

In conclusion, the effect of chemical gradients on the angular momentum transport is negligible in the white dwarf interior, unlike in non-degenerate stars.

\subsubsection{Limitations of our 1D approximation}

As pointed out in Sect. 3.3, our one-dimensional description of rotation works accurately up to about $60 \%$ of Keplerian rotation. In our white dwarf models, however, the fast rotating outer layers exceed this limit (cf. Fig. 13). For instance, in model sequence A6, the outer $19 \%$ in mass rotate faster than $60 \%$ of the Keplerian value when $E_{\text {rot }} /|W|=0.1$. This fast rotating region increases to $27 \%$ in mass when $E_{\text {rot }} /|W|$ reaches 0.14 . This means that our numerical models underestimate the effect of the centrifugal force on the white dwarf structure.

The accreting white dwarf models by Durisen (1977) may be the most appropriate to evaluate the uncertainty due to the underestimation of the centrifugal force in the outer envelope. In his two-dimensional models, the inner dense core with $\rho>0.1 \rho_{\mathrm{c}}$ remains close to spherical, and the outer layers with $\rho<0.1 \rho_{\mathrm{c}}$ start deviating from the spherical symmetry, becoming toroidal. Consequently, the outer envelope is considerably extended, so that the ratio of the polar to the equatorial radius $\left(R_{\mathrm{p}} / R_{\mathrm{e}}\right)$ becomes as small as 0.4 when $E_{\text {rot }} /|W|$ reaches 0.1 .

In our models, the inner slowly rotating core $(\omega<$ $\left.0.6 \omega_{\text {Kepler }}\right)$ has density $\rho \gtrsim 0.1 \rho_{\mathrm{c}}$ in general, and therefore we may conclude that the inner core is accurately described in our calculations, although it could be implicitly affected by the underestimation of the centrifugal force in the outer envelope. However, the major qualitative conclusions of our study are not affected by this uncertainty: accreting white dwarfs will rotate differentially and can grow beyond the canonical Chandrasekhar mass without suffering central carbon ignition, unless they lose angular momentum through secular instabilities (Sect. 5). 


\section{On the final fate of rapidly rotating massive white dwarfs}

A remarkable feature of our models is that the white dwarfs cannot reach central carbon ignition even when their mass becomes significantly larger than the canonical Chandrasekhar mass of $1.4 M_{\odot}$ (Tables 2 and 3). For example, in model sequence $\mathrm{A} 12$, the central density at $M_{\mathrm{WD}}=1.70 M_{\odot}$ is only $3.13 \times 10^{8} \mathrm{~g} / \mathrm{cm}^{3}$ (see Table 3), which is still far from the carbon ignition density (a few $10^{9} \mathrm{~g} / \mathrm{cm}^{3}$ at $T \gtrsim 10^{8} \mathrm{~K}$ ). This is in good agreement with the conclusion of previous studies of rotating white dwarfs (cf. Sect. 3.1) that a white dwarf can be dynamically stable for masses up to $\sim 4.0 M_{\odot}$ if it rotates differentially.

If a rapidly rotating massive white dwarf would not lose angular momentum, it could not produce a Type Ia supernova. However, it is well known that rapidly rotating compact stars become secularly unstable to non-axisymmetric perturbations due to the gravitational wave radiation reaction (Chandrasekhar 1970; Friedman \& Schutz 1978), which is often named CFS (Chandrasekhar, Friedman and Schutz) instability. Two representative modes are believed to be the most important ones for this instability. One is the bar mode, i.e., the $f$-mode with $l=m=2$, of which the restoring force is the buoyancy force. Here, $l$ and $m$ denote the nodal numbers of the spherical harmonics. Although Friedman \& Schutz (1978) found that $f$-modes with a higher $m$ are more susceptible to the CFS instability compared to the case $m=2$, their growth time usually becomes too long to be of astrophysical interest (e.g. Shapiro \& Teukolsky 1983). Hereafter, we will refer to the CFS instability with $m=2$ as the bar-mode instability. Recently it was found that the CFS instability can also excite the $r$-mode, of which the restoring force is the Coriolis force (hereafter, $r$-mode instability, see Andersson \& Kokkotas 2001 for a review). Here again, the mode with $m=l=2$ is most relevant because it gives the smallest time scale for the growth of the instability.

In the following, we discuss the importance of these instabilities for our white dwarf models, and derive implications for the final fate of rapidly rotating massive white dwarfs.

\subsection{Bar-mode instability}

The bar-mode instability can operate when $E_{\text {rot }} /|W|$ exceeds a certain critical value $\left[\left(E_{\mathrm{rot}} /|W|\right)_{\mathrm{bar}, \text { crit }}\right]$ in a rotating star, as studied by many authors (Ostriker \& Tassoul 1969; Ostriker \& Bodenheimer 1973; Durisen 1975b, 1977; Bardeen et al. 1977; Durisen \& Imamura 1981). Although $\left(E_{\mathrm{rot}} /|W|\right)_{\text {bar, crit }}$ is found to be about 0.14 for a wide range of rotation laws and equations of state (e.g. Durisen 1975b; Karino \& Eriguchi 2002), Imamura et al. (1995) showed that it tends to decrease for strong differential rotation. In particular, we note that the rotation law in our models bears a similarity to one of their rotating polytrope models with $n^{\prime}=\infty$ (see Imamura et al. for the definition of $n^{\prime}$ ), in which the spin rate shows a maximum where the gradient in $\omega$ changes its sign, as in our models (Fig. 6). In these models, $\left(E_{\mathrm{rot}} /|W|\right)_{\text {bar, crit }}$ turns out to be as small as 0.09 , which is significantly smaller than the canonical value of 0.14 .

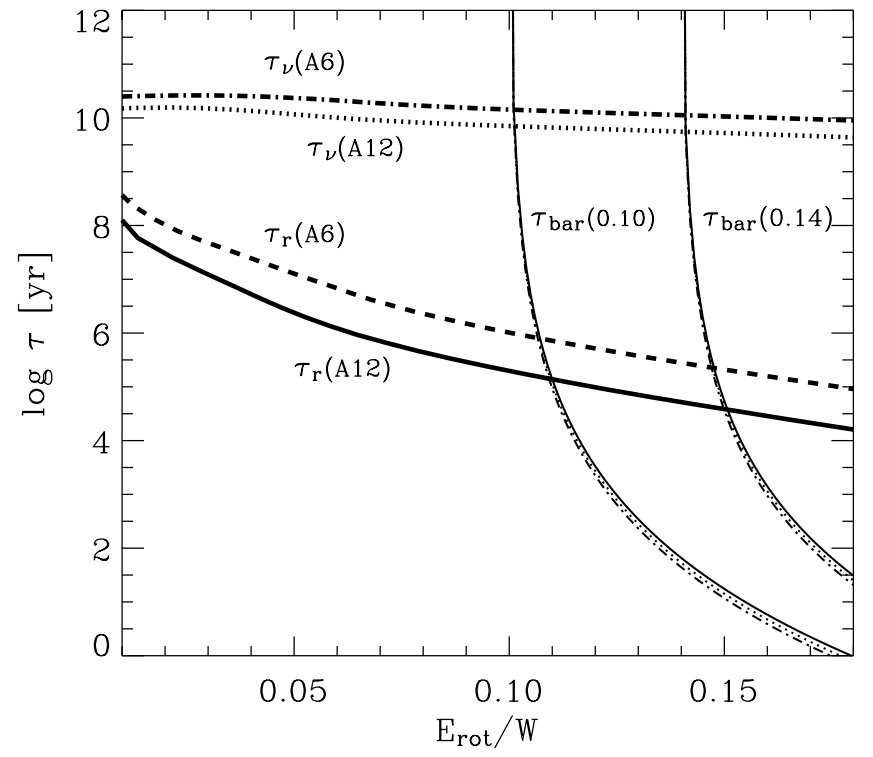

Fig. 14. Growth time scales for the bar-mode instability ( $\tau_{\text {bar }}$; Eq. (20)) as well as for the $r$-mode instability $\left(\tau_{\mathrm{r}}\right.$; Eq. $\left.(21)\right) . \tau_{\text {bar }}$ is plotted as a function of $E_{\mathrm{rot}} /|W|$, with thin lines for 3 different masses: $1.4 M_{\odot}$ (solid line), 1.5 $M_{\odot}$ (dotted line) and 1.6 $M_{\odot}$ (dashed-dotted line). Two different values of $\left(E_{\text {rot }} /|W|\right)_{\text {bar, crit }}(0.1$ and 0.14$)$ for the bar-mode instability are considered, as indicated in the figure. The thick dashed line denotes $\tau_{\mathrm{r}}$ in the models of sequence A6, while the thick solid line is for sequence A12. The thick dashed-dotted and dotted lines give the viscous time scale for sequences A6 and A12 respectively.

According to Chandrasekhar (1970) and Friedman \& Schutz (1975), the growth time of the bar-mode instability in Maclaurin spheroids is given by

$\tau_{\text {bar }} \sim 10^{-8} \frac{R}{c}\left(\frac{R \Omega}{c}\right)^{-6}\left[\frac{E_{\mathrm{rot}}}{|W|}-\left(\frac{E_{\mathrm{rot}}}{|W|}\right)_{\mathrm{bar}, \mathrm{c}}\right]^{-5} \mathrm{~s}$,

for $0<E_{\mathrm{rot}} /|W|-\left(E_{\mathrm{rot}} /|W|\right)_{\mathrm{c}} \ll 1$, where $\Omega$ is the mean angular velocity and $R$ is the radius.

Since we have $E_{\text {rot }} \simeq k^{2} M R^{2} \Omega^{2}$ and $|W| \simeq G M^{2} / R$ (where $k$ is the dimensionless radius of gyration), the growth time of the instability can be rewritten as (cf. Friedman \& Schutz 1975; Hayashi et al. 1998)

$$
\begin{aligned}
\tau_{\text {bar }} \sim & 1.6\left(\frac{R}{0.01 R_{\odot}}\right)^{4}\left(\frac{M}{M_{\odot}}\right)^{-3}\left(\frac{E_{\mathrm{rot}}}{|W|}\right)^{-3} \\
& \times\left[\frac{E_{\mathrm{rot}}}{|W|}-\left(\frac{E_{\mathrm{rot}}}{|W|}\right)\right]_{\mathrm{c}}^{-5} \mathrm{~s},
\end{aligned}
$$

where $k^{2}=0.4$ has been used. Although our white dwarf models deviate significantly from the Maclaurin spheroids, this approximation may give an order of magnitude estimate for the growth time scale of the bar-mode instability.

Figure 14 shows $\tau_{\text {bar }}$ as a function of $E_{\text {rot }} /|W|$ for four different masses as indicated in the figure caption. Here, $R=$ $0.01 R_{\odot}$ is assumed since the mean radii of our white dwarf models do not differ much from $0.01 R_{\odot}$ for all $E_{\text {rot }} /|W|$ values. Two different values for $\left(E_{\text {rot }} /|W|\right)_{\text {bar, crit }}$, i.e., 0.1 and 0.14 are considered in the figure. The growth time scale of the barmode instability is a sensitive function of $E_{\text {rot }} /|W|$, while it 
does not change much for different white dwarf masses: for $\left(E_{\text {rot }} /|W|\right)_{\text {bar, crit }}=0.10, \tau_{\text {bar }}$ is as large as $10^{10} \mathrm{yr}$ at $E_{\text {rot }} /|W| \simeq$ $\left(E_{\text {rot }} /|W|\right)_{\text {bar, crit }}$, but it drops to $10^{4} \mathrm{yr}$ when $E_{\text {rot }} /|W| \simeq 0.12$. Furthermore, $\tau_{\text {bar }}$ becomes only about 10 yrs when $E_{\text {rot }} /|W|$ approaches 0.15 .

Durisen (1977) found that the angular momentum loss time scale is comparable to the growth time scale of the instability $\left(\tau_{J \text {,bar }} \simeq \tau_{\text {bar }}\right)$, and therefore we may conclude that the bar-mode instability can be an efficient mechanism to remove angular momentum for white dwarfs with $E_{\text {rot }} /|W|>$ $\left(E_{\text {rot }} /|W|\right)_{\text {bar, crit }}$, as long as it is not suppressed by turbulent motions (see Sect. 5.3).

\section{2. r-mode instability}

Recently, Andersson (1998) and Friedman \& Morsink (1998) have found that $r$-modes in all rotating inviscid stars are unstable to the CFS instability. The possibility of gravitational wave radiation from fast rotating white dwarfs due to the $r$-mode instability was also discussed by Hiscock (1998), Andersson et al. (1999) and Lindblom (1999). Lindblom (1999) gives the growth time scale for this instability with $m=l=2$ as

$$
\frac{1}{\tau_{\mathrm{r}}}=\frac{2 \pi}{25}\left(\frac{4}{3}\right)^{8} \frac{G}{c} \int_{0}^{R} \rho\left(\frac{r \Omega}{c}\right)^{6} \mathrm{~d} r .
$$

This time scale is plotted in Fig. 14 for two model sequences (A6 and A12). Interestingly, we find that the $r$-mode instability becomes important when $E_{\text {rot }} /|W| \lesssim\left(E_{\text {rot }} /|W|\right)_{\text {bar, crit }}$, compared to the bar-mode instability. The sensitivity of the time scale $\tau_{\mathrm{r}}$ to $E_{\mathrm{rot}} /|W|$ is much weaker than that of $\tau_{\mathrm{bar}}$, and $\tau_{\mathrm{r}}$ remains in the range $10^{5} \ldots 10^{8} \mathrm{yr}$ for $E_{\mathrm{rot}} /|W|<$ $\left(E_{\text {rot }} /|W|\right)_{\text {bar, crit }}$. Note that $\tau_{\mathrm{r}}$ in our models has much smaller values than in Lindblom (1999), who found $\tau_{\mathrm{r}}$ to be larger than $6 \times 10^{9} \mathrm{yr}$ in the observed DQ Her objects. This difference is mainly due to the fact that our white dwarf models are rotating differentially, with the outer layer rotating faster than the inner core. More precise estimates of $\tau_{\mathrm{r}}$ require, of course, a multidimensional study, which is beyond the scope of this paper. Nevertheless, the present estimates indicate that the $r$-mode instability might be a promising mechanism for the removal of angular momentum in a differentially rotating massive white dwarf within an interestingly short time scale $\left(\lesssim 10^{8} \mathrm{yr}\right)$, when $E_{\text {rot }} /|W|<\left(E_{\text {rot }} /|W|\right)_{\text {bar, crit }}$.

\subsection{Implications for the final fate of accreting white dwarfs}

The previous sections imply that our differentially rotating massive white dwarfs can lose angular momentum via the bar-mode instability if $E_{\text {rot }} /|W| \gtrsim\left(E_{\text {rot }} /|W|\right)_{\text {bar, crit }}$, and via the $r$-mode instability if $E_{\text {rot }} /|W| \lesssim\left(E_{\text {rot }} /|W|\right)_{\text {bar, crit }}$. These instabilities would remove angular momentum from the outer layers of the white dwarfs - as shown in the numerical simulations of rotating neutron stars (Lindblom et al. 2002) - where most of the white dwarf angular momentum is located in our models.

Although the CFS instability is likely suppressed in the presence of strong viscosity for both the $r$-mode and the bar mode (e.g. Andersson \& Kokkotas 2001), Imamura et al. (1995) suggest that secularly unstable modes may not necessarily be damped in the presence of a large effective viscosity due to turbulence. Given this uncertainty, we consider both cases, i.e., we assume strong damping of secular instabilities due to turbulence induced by the shear instability as Case I, and a persistence of secular modes even when the shear instability is present, where the CFS instability is only affected by the microscopic viscosity (Case II).

\subsubsection{Case I}

If the unstable CFS modes are damped by turbulence, an accreting white dwarf will not experience any angular momentum loss due to the CFS instability during the mass accretion phase in which the dynamical and secular shear instability persist throughout the white dwarf interior (see Sect. 4.2), no matter how large $E_{\mathrm{rot}} /|W|$ becomes. Once the mass accretion ceases, the angular momentum redistribution in the white dwarf will continue until the degree of the differential rotation becomes weak enough for the shear instability to disappear. Once the fluid motion in the white dwarf becomes laminar, only the electron and ion viscosities will contribute to the viscous friction.

The time scale of viscous dissipation $\left(\tau_{v}\right)$ through the electron and ion viscosity is plotted in Fig. 14 for model sequences A6 and A10. Here $\tau_{v}$ is calculated following Eq. (4.2) in Lindblom (1999). It is found that $\tau_{v}$ is far larger than $\tau_{\mathrm{r}}$ and $\tau_{\text {bar }}$ (except for $\tau_{\text {bar }}$ at $\left.E_{\text {rot }} /|W| \simeq\left(E_{\text {rot }} /|W|\right)_{\text {bar, crit }}\right)$. This implies that the unstable modes of the CFS instability will not be damped by the microscopic viscosity before they grow to a dynamically meaningful level, once the shear instability has decayed.

If the white dwarf mass has grown to $M_{\mathrm{WD}} \gtrsim 1.4 M_{\odot}$ by the end of the mass accretion phase, carbon ignition at the white dwarf center will be delayed as follows. After the mass accretion stops, the white dwarf will evolve without losing angular momentum until the shear instability decays. When the white dwarf interior becomes laminar, the white dwarf will begin to lose the angular momentum either by the bar-mode instability if $E_{\mathrm{rot}} /|W|>\left(E_{\mathrm{rot}} /|W|\right)_{\mathrm{bar}, \text { crit }}$, or by the $r$-mode instability if $E_{\text {rot }} /|W| \lesssim\left(E_{\text {rot }} /|W|\right)_{\text {bar, crit }}$. The core density will increase as it loses angular momentum and eventually carbon will ignite at the center.

Can such a white dwarf results in a Type Ia supernova? It depends on how much time there is from the halt of mass accretion to carbon ignition, the time scale to which we will refer as $\tau_{\text {delay }}$. Nomoto \& Kondo (1993) found that if the core of a white dwarf has solidified, core carbon ignition is likely to result in a collapse rather than an explosion. I.e., to obtain an SN Ia, carbon ignition should occur before the white dwarf core is crystallized.

In an attempt to estimate $\tau_{\text {delay }}$, we have selected a model in which the white dwarf has grown in mass to $1.50 M_{\odot}$ in sequence $\mathrm{A} 12$, and let it evolve without further mass accretion. The evolution of the angular velocity in the white dwarf model is shown in Fig. 15. The initial model of this calculation is characterized by $T_{\mathrm{c}}=1.37 \times 10^{8} \mathrm{~K}, \rho_{\mathrm{c}}=1.87 \times 10^{8} \mathrm{~g} / \mathrm{cm}^{3}$ and 

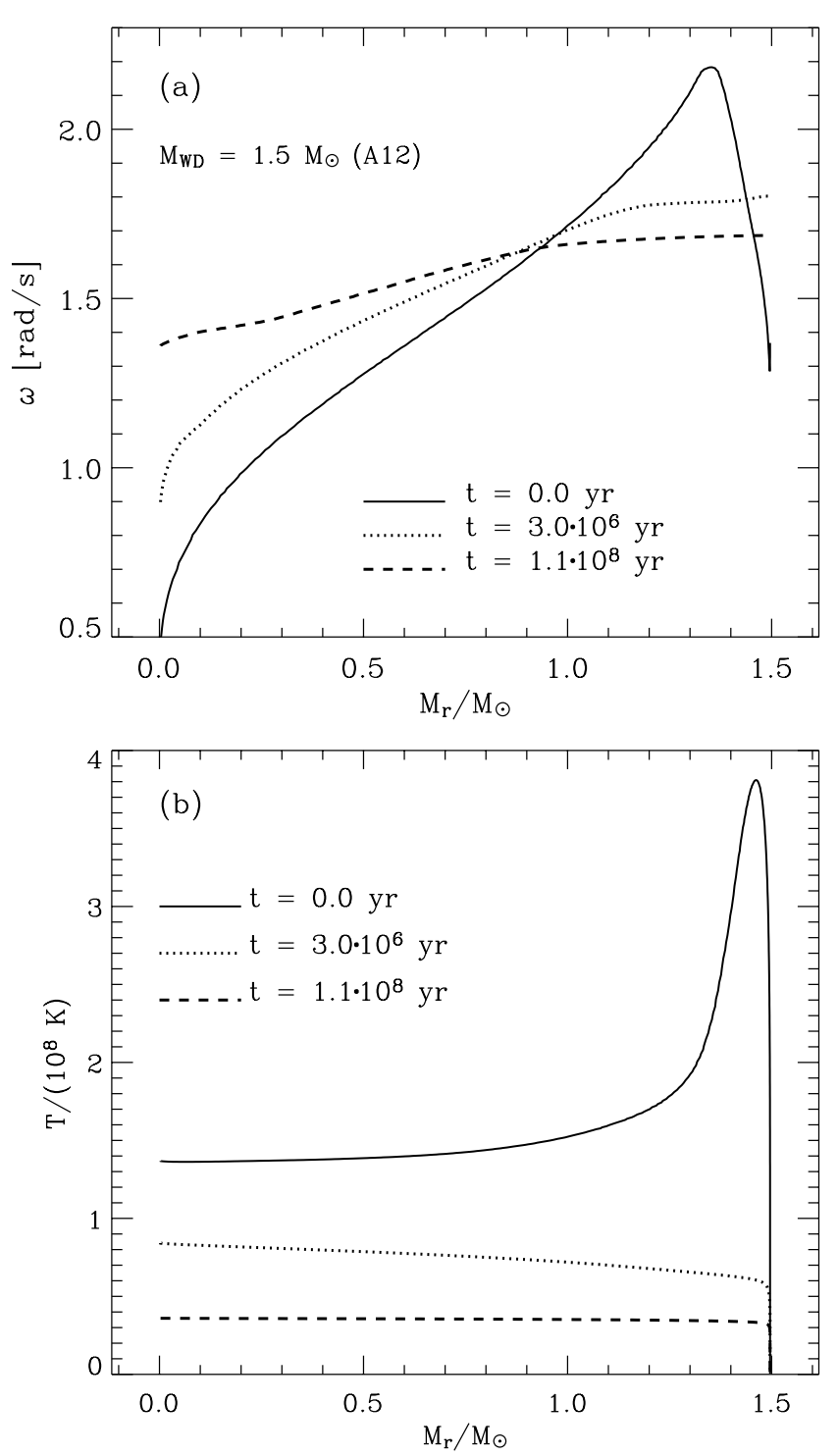

Fig. 15. Evolution of the angular velocity in a), and temperature in b), of a rapidly rotating $1.50 M_{\odot}$ white dwarf after the mass accretion stops. The initial model at $t=0$ has been taken from model sequence A12.

$E_{\text {rot }} /|W|=1.11$. As the white dwarf cools, the buoyancy force in the white dwarf interior becomes weaker and thus the dynamical shear instability continues to operate in the core even though the degree of differential rotation becomes continuously smaller. Only when $t \simeq 1.1 \times 10^{8}$ yr does the shear strength become so weak that the shear instability finally decays. The central temperature and density at this moment are $4 \times 10^{7} \mathrm{~K}$ and $1.57 \times 10^{8} \mathrm{~g} / \mathrm{cm}^{3}$ respectively. This example indicates that the CFS instability can begin to operate about $10^{8}$ yr after the end of the mass accretion. Which instability mode is responsible for the following angular momentum loss depends on the value of $\left(E_{\text {rot }} /|W|\right)_{\text {bar, crit }}$ of the white dwarf configuration at this point.

If the angular momentum loss time scale is short enough, (i.e., $\tau_{J} \lesssim 10^{7}$ ), the white dwarf may end as a Type Ia supernova, since the central temperature is still relatively high, and it will increase by the contraction of the core as the white dwarf loses angular momentum. Piersanti et al. (2003) showed that an initially cool Chandrasekhar mass white dwarf with $T_{\mathrm{c}} \simeq 5.6 \times 10^{7} \mathrm{~K}$ is heated to $T_{\mathrm{c}} \sim 10^{8} \mathrm{~K}$ if the white dwarf loses angular momentum on a time scale of $10^{4} \ldots 10^{7} \mathrm{yr}$.

In the example given above, $E_{\text {rot }} /|W|$ decreases from 0.11 to 0.10 during the evolution. If $\left(E_{\text {rot }} /|W|\right)_{\text {bar, crit }}$ at this point is less than 0.1 , the bar-mode instability will remove angular momentum in $\tau_{J \text {,bar }}$, which may be short enough (i.e., $\lesssim 10^{7} \mathrm{yr}$ ) to produce an SN Ia. On the other hand, if $\left(E_{\mathrm{rot}} /|W|\right)_{\mathrm{bar}}$, crit is larger than 0.1 at the given moment, the $r$-mode instability will become dominant. The value of $\tau_{\mathrm{r}}$ at this point is about $2 \times$ $10^{5} \mathrm{yr}$. If the time scale for the following angular momentum loss is of the order of $\tau_{\mathrm{r}}$, the white dwarf will safely end as an SN Ia.

Although this discussion is somewhat speculative, it may suggest an interesting implication for the masses of white dwarfs in SNe Ia explosions. If the CFS instability is suppressed by the shear instability, white dwarfs can grow in principle until they become dynamically unstable $\left(E_{\text {rot }} /|W| \gtrsim 0.27\right)$ without becoming secularly unstable. In most close binary systems, the mass accretion will stop before reaching this point, given that the mass at $E_{\text {rot }} /|W|=0.18\left(M_{0.18}\right)$ in our models can be already as high as $1.45 M_{\odot}-1.75 M_{\odot}$ (Table 3). Therefore, the upper limit for the white dwarf mass at the moment of the supernova explosion $\left(M_{\mathrm{SN}}\right)$ is determined by the maximum possible mass a white dwarf can achieve by mass accretion, $\left(M_{\max }\right)$. The lower limit for $M_{\mathrm{SN}}$ is simply $1.4 M_{\odot}$. I.e., the white dwarf mass at the moment of supernova explosion will be between $1.4 M_{\odot}$ and $M_{\max }\left(1.4 M_{\odot} \lesssim M_{\mathrm{SN}} \lesssim M_{\max }\right)$.

The lower limit for $M_{\mathrm{SN}}\left(1.4 M_{\odot}\right)$ cannot be different for different initial masses. However, the upper limit $\left(M_{\max }\right)$ may be systematically larger for higher initial masses. Langer et al. (2000), found that $M_{\max }$ is larger for a higher $M_{\text {init }}$ in main sequence star + white dwarf binary systems. Furthermore, $M_{\max }$ is expected to show a dependence on the metallicity of the mass donor. Langer et al. (2000) showed that $M_{\max }$ for main sequence star + white dwarf binary systems decreases for lower metallicity. This implies a higher probability to obtain massive exploding white dwarfs for higher metallicity. If more massive white dwarfs gave brighter SNe Ia as Fisher et al. (1999) speculate, the brightest SNe Ia observed at low metallicity may be dimmer than those at higher metallicity, while the luminosity of the dimmest may not be much different (unless affected by the CO ratio; Umeda et al. 1999; Höflich et al. 2000).

\subsubsection{Case II}

If the CFS instability is not affected by the shear instability, an accreting white dwarf will start losing angular momentum when $\tau_{\mathrm{r}}$ or $\tau_{\text {bar }}$ becomes smaller than the accretion time scale $\left(\tau_{\mathrm{acc}} \simeq \Delta M / \dot{M}\right)$.

If we consider sequence A6 as an example, $\tau_{\mathrm{r}}$ becomes comparable to $\tau_{\text {acc }}$ only when $E_{\text {rot }} /|W| \simeq 0.14$. Therefore, if we assume $\left(E_{\text {rot }} /|W|\right)_{\text {bar, crit }}=0.1$, the bar-mode instability will dominate before the $r$-mode instability becomes important. Once $E_{\text {rot }} /|W|$ becomes larger than 0.1 , the white dwarf will lose angular momentum via the bar-mode instability while gaining angular momentum continuously from the accreted 
matter. It is likely that $E_{\text {rot }} /|W|$ will not change much after $\tau_{\text {bar }} \approx \tau_{\text {acc }}$ is obtained. This means that $E_{\text {rot }} /|W|$ cannot increase much from the critical value of 0.1 , since $\tau_{\text {bar }}$ drops rapidly as it deviates from $\left(E_{\text {rot }} /|W|\right)_{\text {bar, crit }}$ (Fig. 14). If mass accretion continues, the white dwarf will finally reach carbon ignition at $M_{\mathrm{WD}} \simeq 1.76 M_{\odot}$, according to the results of sequences $\mathrm{C} 9 \ldots \mathrm{C} 12$, where carbon ignites at the center when $M_{\mathrm{WD}} \simeq 1.76 M_{\odot}$, with $E_{\text {rot }} /|W| \simeq 0.11$ (see also Sect. 7). If mass accretion stops before carbon ignition is reached but after the white dwarf mass has grown beyond $1.4 M_{\odot}$, carbon ignition will be delayed until the white dwarf loses enough angular momentum.

In summary, we can expect that even in Case II the mass of exploding white dwarfs will show a significant diversity, as in Case I. The lower limit for $M_{\mathrm{SN}}$ should be $\sim 1.4 M_{\odot}$ in both cases, but the upper limit may be either the critical mass for carbon ignition at that $E_{\mathrm{rot}} /|W|$ which gives $\tau_{\mathrm{J}} \approx \tau_{\mathrm{acc}}$, or the maximum possible achievable mass by mass accretion.

\subsubsection{Other uncertainties}

In reality, the mass transfer rate in a close binary system does not remain constant. For instance, in a binary system consisting of a main sequence star + a white dwarf, where thermally unstable mass transfer occurs, the mass transfer rate increases rapidly from the onset of the mass transfer, followed by slow decrease to the point that the nuclear shell burning cannot be stable any more (Langer et al. 2000). Although shell sources may be more stable with rotation (Yoon et al. 2004), the white dwarf will experience strong shell flashes if the mass accretion rate decreases below $10^{-7} M_{\odot} / \mathrm{yr}$. This may cause a significant loss of mass, which may even decrease the white dwarf mass. This may also lead to a removal of angular momentum from the white dwarf (Livio \& Pringle 1998). Effects of magnetic fields, if they are strongly amplified due to the differential rotation (Spruit 2002), may also serve to brake the white dwarf by magnetic torques and/or by magnetic dipole radiation. These possibilities will be the subject of further studies.

\section{Implications for the SNe la diversity}

We note that for all considered circumstances, the effects of rotation in accreting white dwarfs offer the possibility of SN Ia explosions from white dwarfs more massive than $\sim 1.4 M_{\odot}$ (hereafter designated as super-Chandrasekhar mass). The observational signatures of super-Chandrasekhar mass explosions are currently difficult to predict. The peak brightness values and light curves of SNe Ia might depend sensitively on the ignition conditions such as the $\mathrm{CO}$ ratio, the density at the moment of carbon ignition, the speed of the deflagration front, the core rotation rate, and possibly the transition density at which the deflagration turns into a detonation (e.g. Khokholov 1991a, 1991b; Höflich \& Khokhlov 1996; Niemeyer \& Woosley 1997; Höflich et al. 1998; Iwamoto et al. 1999; Umeda et al. 1999; Hillebrandt \& Niemeyer 2000; Woosley et al. 2003).

If the ignition conditions are not strongly affected by the mass of the exploding white dwarf, it is likely that a more massive white dwarf gives a brighter SN Ia, since more fuel to produce ${ }^{56} \mathrm{Ni}$ can be provided. For instance, progenitors with a super-Chandrasekhar mass are often invoked to explain such an anomalous SN Ia as SN 1991T. Fisher et al. (1999) note that the mass of ${ }^{56} \mathrm{Ni}$ produced from the explosion models of a Chandrasekhar mass white dwarf remains smaller than about $0.9 M_{\odot}$ even in the case where a pure detonation is considered (e.g. Höflich \& Khokhlov 1996). Although the nickel masses derived from the luminosities of normal SNe Ia are constrained to $0.4 \ldots 0.8 M_{\odot}$ (Leibundgut 2000), the existing models fail to explain such a peculiar SN Ia as SN 1991T, whose brightness implies the production of about $1.0 M_{\odot}$ of ${ }^{56} \mathrm{Ni}$. The fact that explosion models with the canonical Chandrasekhar mass could not explain the peculiarity of SN 1991T suggests that it is worthwhile to investigate the possible outcome of superChandrasekhar mass explosions, even if we cannot exclude the possibility that the luminosity of $1991 \mathrm{~T}$ is biased by the uncertainty in determining the distance of its host galaxy (Hanato et al. 2002).

On the other hand, Fisher et al. (1999) suggested the explosion of a super-Chandrasekhar mass white dwarf from the merger of double $\mathrm{CO}$ white dwarfs as explanation of the overluminosity found in SN 1991T. We note that differentially rotating single degenerate progenitors may be a more natural explanation for the super-Chandrasekhar mass scenario than double degenerate mergers, which fail to produce SNe Ia in numerical models due to the off-center carbon ignition induced by the fast mass accretion with $\dot{M} \gtrsim 10^{-5} M_{\odot} /$ yr (Saio \& Nomoto 1985 , 1998).

Rotation also has implications for the polarization of $\mathrm{SNe}$ Ia. According to our results, the rotation velocity of exploding white dwarfs may strongly depend on the history of angular momentum loss via gravitational wave radiation. The more angular momentum is lost, the slower the white dwarf rotation will be, which means that the polarization strength in SNe Ia may show significant diversity. Some white dwarfs may have a chance to result in an SN Ia while they are rotating very rapidly, as in sequences $\mathrm{C} 9, \mathrm{C} 10, \mathrm{C} 11$ and $\mathrm{C} 12$, where carbon ignition occurs at the white dwarf center when $M_{\mathrm{WD}} \simeq 1.76 M_{\odot}$ and $E_{\text {rot }} /|W| \simeq 0.11$. Such exploding white dwarfs will show strong features of asphericity in the explosion, which might give a plausible explanation for the polarization observations in SN 1999 by (Howell et al. 2001) and SN 2001 el (Wang et al. 2003; Kasen et al. 2003).

\section{Pre-explosion conditions of a fast rotating white dwarf}

Rotation may have interesting consequences for the supernova explosion itself. Figure 16 shows the physical properties of the white dwarf model of sequence C9 when $M_{\mathrm{WD}}=1.76 M_{\odot}$, where the central temperature and density reach $5.5 \times 10^{8} \mathrm{~K}$ and $2.2 \times 10^{9} \mathrm{~g} / \mathrm{cm}^{3}$, respectively. The central region $(0.0 \lesssim$ $\left.M_{\mathrm{r}} \lesssim 0.6 M_{\odot}\right)$ is found to be convectively unstable due to carbon burning, rotating rigidly due to the convective angular momentum redistribution. A thermonuclear runaway is expected to develop when the central temperature reaches a few times $10^{9} \mathrm{~K}$. The convective core will be more extended by then, spinning up the central region further. In the model shown 

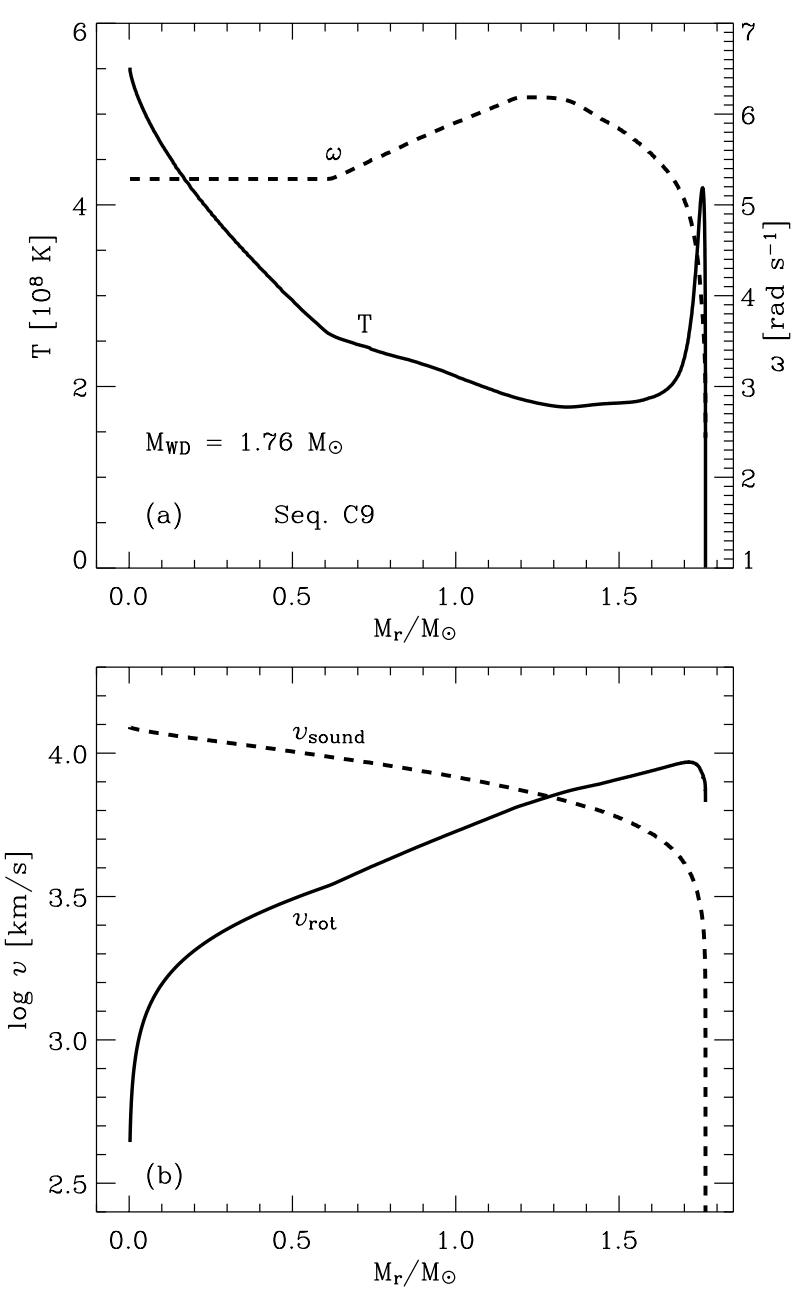

Fig. 16. a) Temperature (solid line) and angular velocity (dashed line) as a function of the mass coordinate in the last computed model of sequence C9. b) Equatorial rotational velocity (solid line) for the same model as in a) as a function of the mass coordinate. The dashed line denotes the sound speed as a function of the mass coordinate.

in Fig. 16, the central region rotates with $v_{\text {rot }} \simeq 1000 \mathrm{~km} \mathrm{~s}^{-1}$, which is well above the convective velocity $\left(\sim 100 \mathrm{~km} \mathrm{~s}^{-1}\right.$; e.g. Höflich \& Stein 2002) and somewhat larger than the expected initial velocity of the deflagration front $\left(v_{\text {def,init }} \simeq 0.03 v_{\text {sound }}\right.$, e.g. Nomoto et al. 1984). The equatorial rotation velocity increases sharply from the center to the edge of the convective layer $\left(M_{\mathrm{r}}=0.6 M_{\odot}\right)$, by a factor of 10 .

This fast rotation in the central carbon burning core may affect the explosion as follows. Numerous studies indicate that the combustion front develops large scale turbulent motions, especially due to the Rayleigh-Taylor instability (e.g. Reinecke et al. 2002; Gamezo et al. 2003, and references therein). It is a crucial question whether this turbulence can induce a detonation (Khokhlov 1991a,b; Niemeyer \& Woosley 1997; Khokhlov et al. 1997). Although pure deflagration models show a good agreement with observations (Nomoto et al. 1984; Höflich \& Khokhlov 1996), recent three-dimensional calculations show that large amounts of unburned and partially burnt materials are left even at the center (Reinecke et al. 2002; Gamezo et al. 2003), which is not supported by observations (however, see Baron et al. 2003). This problem would disappear if a detonation were triggered by the turbulent deflagration (“delayed detonation", Khokhlov 1991a). Furthermore, delayed detonation models are shown to reproduce the light curves and spectra of well observed Type Ia SNe (Höflich \& Khokhlov 1996; Höflich et al. 1996). A study of the nucleosynthesis output of SNe Ia by Iwamoto et al. (1999) also favors the delayed detonation scenario.

Several authors conclude that the probability of triggering a detonation can be enhanced if the turbulence is strong enough to form a thick mixed region of unburned and burning materials with a constant temperature gradient (Khokhlov 1991b; Niemeyer \& Woosley 1997; Khokhlov et al. 1997; Lisewski et al. 2000). However, no robust mechanism for the formation of such a strong turbulence has yet been suggested. (Niemeyer 1999).

A rapidly rotating white dwarf like the one shown in Fig. 16 will be spun down due to expansion once the thermal runaway occurs, as the deflagration wave propagates. However, the outer layers will still rotate rapidly at $v_{\text {rot }} \sim 10^{3} \mathrm{~km} \mathrm{~s}^{-1}$. Lisewski et al. (2000) show that strong turbulence, i.e. $v_{\text {turb }} \approx 10^{3} \mathrm{~km} \mathrm{~s}^{-1}$, is necessary for a detonation to form successfully. Since the rotation velocity in the outer region will be of this order, the shear motion may provide enough kinetic energy for the turbulence intensity to satisfy the condition for a transition of the deflagration into a detonation, which might not be possible in the nonrotating case since the maximum value for $v_{\text {turb }}$ is $\sim 10^{2} \mathrm{~km} \mathrm{~s}^{-1}$ in the Rayleigh-Taylor-driven turbulence. Alternatively, the fast rotation in the outer region can tear the turbulent deflagration front and enhance the nuclear burning surface, which may be another way to trigger a detonation (Höflich 2003, private communication). Future multi-dimensional calculations are required to test the validity of these scenarios.

\section{Gravitational wave radiation in pre-explosion white dwarfs?}

Rotation may have another important and exciting observational consequence. Our results indicate that the white dwarfs in SN Ia progenitor systems may emit gravitational wave radiation (GWR). Therefore, if we could observe GWR preceding an SN Ia event, this would provide strong evidence for the scenarios outlined in Sect. 5.

The GWR due to the CFS bar-mode instability may be much stronger than the $r$-mode signal, since perturbations due to the bar mode involve larger density changes than those due to the $r$-mode (e.g. Andersson \& Kokkotas 2001). Fryer et al. (2002) derive the gravitational wave amplitude observed at Earth from a source which undergoes the bar-mode instability at a distance $d$ as

$h_{\mathrm{bar}}=\left(\frac{32}{45}\right)^{1 / 2} \frac{G}{c^{4}} \frac{M R^{2} \Omega^{2}}{d}$,

where $R$ denotes the equatorial radius of the source, $M$ the mass and $\Omega$ the angular velocity. The frequency of the gravitational waves $\left(f_{\mathrm{GW}}\right)$ due to the bar-mode instability is twice the rotation frequency of the source. We estimate $h_{\mathrm{bar}}$ and $f_{\mathrm{GW}}$ for 
Table 4. Expected properties of the gravitational waves from the selected accreting white dwarfs.

\begin{tabular}{rcccc}
\hline \hline No. & $M$ & $E_{\text {rot }} /|W|$ & $\begin{array}{c}h_{\text {bar }} \text { at } 10 \mathrm{Mpc} \\
10^{-24}\end{array}$ & $\begin{array}{c}f_{\mathrm{GW}} \\
\mathrm{Hz}\end{array}$ \\
\hline $\mathrm{A} 2$ & 1.18 & 0.10 & 1.7 & 0.22 \\
$\mathrm{~A} 6$ & 1.30 & 0.10 & 2.9 & 0.32 \\
$\mathrm{~A} 10$ & 1.42 & 0.10 & 4.3 & 0.46 \\
$\mathrm{~A} 2$ & 1.34 & 0.14 & 3.7 & 0.32 \\
$\mathrm{~A} 6$ & 1.44 & 0.14 & 5.0 & 0.43 \\
$\mathrm{~A} 10$ & 1.55 & 0.14 & 7.5 & 0.62 \\
\hline
\end{tabular}

selected white dwarf models in Table 4. For these calculations, we used mean values for $\Omega$ and $R$ given in Tables 2 and 3 .

Table 4 indicates that $f_{\mathrm{GW}}$ is within the range of $0.1 \ldots 1.0 \mathrm{~Hz}$. Observation of GWs with such frequencies may be performed by low frequency gravitational wave detectors such as LISA, which will cover the range $10^{-4}-1.0 \mathrm{~Hz}$ (e.g. Cutler \& Thorne 2002). The expected strength of the gravitational wave signal is about $10^{-24}$ at $d=10 \mathrm{Mpc}$. Hiscock (1998) estimated the strength of GWs due to the $r$-mode instability from white dwarfs in the observed DQ Her systems as $h \sim 10^{-23}$. His calculations show that this strength is well above the detection limit of the LISA interferometer. Therefore, the results shown in Table 4 imply that SNe Ia progenitors in nearby galaxies could be within the observable range. Given that GWs from SN Ia progenitors will be emitted over a secular time, and that SNe Ia are observed with a relatively high rate of $\sim 3 \times 10^{-3} \mathrm{yr}^{-1}$ per galaxy, the probability to detect the GW signal may be considerable.

Even if a white dwarf would not reach the bar-mode instability at $\left(E_{\text {rot }} /|W|\right)_{\text {bar, crit }}$, it might still emit gravitational waves via the $r$-mode instability. Although their strength is rather unclear and may be much weaker than that of the bar mode, they may still be detectable if the source is close enough.

\section{Conclusion}

We summarize the results of this paper as follows.

1. The role of the Eddington-Sweet circulation, the GSF instability and the shear instability for the transport of angular momentum in non-magnetized white dwarfs has been investigated (Sect. 2). Although the Eddington-Sweet circulation and the GSF instability are important for the redistribution of angular momentum in the non-degenerate envelope, their importance is small in the degenerate core compared to the shear instability. The secular shear instability cannot operate in the strongly degenerate core, since the thermal diffusion time becomes longer than the turbulent viscous time for densities higher than a critical value (i.e., $\rho \gtrsim 10^{6} \ldots 10^{7} \mathrm{~g} / \mathrm{cm}^{3}$, Fig. 2). On the other hand, the criterion for the dynamical shear instability is significantly relaxed for higher density because the buoyancy force becomes weaker with stronger degeneracy (Fig. 1). As a result, the degenerate inner core is dominated by the dynamical shear instability in accreting white dwarfs, as shown in Sect. 4.2.
2. We have followed the redistribution of the angular momentum in accreting white dwarfs by the above mentioned processes (Sect. 4.2). We find that accreting white dwarfs do not rotate rigidly but differentially, throughout their evolution, for the considered accretion rates $(\dot{M}=3 \ldots 10 \times$ $\left.10^{-7} M_{\odot} / \mathrm{yr}\right)$. In the degenerate core, once the shear factor decreases to the threshold value for the onset of the dynamical shear instability, the time scale for further angular momentum transport becomes larger than the accretion time scale. Accordingly, strong differential rotation is retained in the inner core with a shear strength near the threshold value for the dynamical shear instability (Fig. 7).

3. Accreting white dwarfs, as they rotate differentially, may not reach central carbon ignition even when they grow beyond the canonical Chandrasekhar limit of $\sim 1.4 M_{\odot}$. This is in accordance with previously obtained results based on other methods (Ostriker \& Bodenheimer 1968, 1973; Durisen 1975b; Durisen \& Imamura 1981). A secular instability to gravitational wave radiation through the $r$-mode or the bar mode may be important for determining the final fate of accreting white dwarfs. The masses of exploding white dwarfs are expected to vary in the range of from the canonical Chandrasekhar mass $\left(\sim 1.4 M_{\odot}\right)$ to the maximum possible mass that the white dwarf can achieve by mass accretion in a binary system ( 2.0 $M_{\odot}$, Langer et al. 2000). This may have consequences for the diversity in the brightness and polarization of SNe Ia.

4. Fast rotation in the white dwarf core may change the supernova explosion since it can affect the evolution of the turbulent nuclear burning flames by providing a large amount of turbulent kinetic energy and/or by enhancing the burning surface significantly. It needs to be clarified whether this may be a plausible mechanism to induce the transition from deflagration to detonation.

5. White dwarfs that have accreted enough angular momentum may be detectable sources of gravitational waves in the near future. Our models show that these will emit gravitational waves with frequencies of $0.1-1.0 \mathrm{~Hz}$. Space-based interferometric gravitational wave detectors such as LISA could observe such signals from rapidly rotating SNe Ia progenitors in nearby galaxies.

Acknowledgements. We are grateful to Axel Bonačić, Peter Höflich and Philipp Podsiadlowski for many useful discussions. This research has been supported in part by the Netherlands Organization for Scientific Research (NWO).

\section{References}

Anand, S. P. S. 1968, ApJ, 153, 135

Andersson, N. 1998, ApJ, 502, 708

Andersson, N., Kokkotas, K. D., \& Stergioulas, N. 1999, ApJ, 516, 307

Andersson, N., \& Kokkotas, K. D. 2001, Int. J. Mode. Phys. D, 10, 381

Bardeen, J. M., Friedman, J. L., Schutz, B. F., \& Sorkin, R. 1977, ApJ, 217, L49

Baron, E., Lentz, E. J., \& Hauschildt, P. H. 2003, ApJ, 588, 29 
Blinnikov, S. I., Dunina-Barkovskaya, N. G., \& Nadyozhin, D. K. 1996, ApJS, 106, 171

Branch, D. 1998, ARA\&A, 36, 17

Branch, D. 2001, PASP, 113, 17

Canuto, V. 1970, ApJ, 159, 641

Cassisi, S., Iben, I. Jr., \& Tornamé, A. 1998, ApJ, 496, 376

Chandrasekhar, S. 1970, Phys. Rev. L, 24, 611

Cutler, C., \& Thorne, K. 2002 [gr-qc/0204090]

Durisen, R. H. 1973a, ApJ, 183, 205

Durisen, R. H. 1973b, ApJ, 183, 215

Durisen, R. H. 1975a, ApJ, 195, 488

Durisen, R. H. 1975b, ApJ, 199, 179

Durisen, R. H. 1977, ApJ, 213, 145

Durisen, R. H., \& Imamura, J. N. 1981, ApJ, 243, 612

Endal, A. S., \& Sofia, S. 1976, ApJ, 210, 184

Fisher, A., Branch, D., Hatano, K., \& Baron, E. 1999, MNRAS, 304, 67

Fliegner, J. 1993, Diplomarbeit, University of Göttingen

Fricke, K. 1968, ZfAP, 68, 317

Friedman, J. L., \& Morsink, S. M. 1998, ApJ, 502, 714

Friedman, J. L., \& Schutz, B. 1975, ApJ, 199, L157

Friedman, J. L., \& Schutz, B. 1978, ApJ, 222, 281

Fryer, C. L., Holz, D. E., \& Hughes, S. 2002, ApJ, 565, 430

Fujimoto, M. Y., \& Sugimoto, D. 1982, ApJ, 257, 291

Gamezo, V. N., Khokhlov, A. M., Oran, E. S., et al. 2003, Science, 299, 77

Goldreich, P., \& Schubert, G. 1967, ApJ, 150, 571

Hachisu, I. 1986, ApJS, 61, 479

Hachisu, I., Kato, M., Nomoto, K., \& Umeda, H. 1999, ApJ, 519, 314

Hamuy, M., Phillips, M. M., Suntzeff, N. B., et al. 1996, ApJ, 519, 314

Hanato, K., Branch, D., Qui, Y. L., et al. 2002, New Astron., 7, 441

Hayashi, A., Eriguchi, Y., \& Mashimoto, M. 1998, ApJ, 492, 286

Heber, U., Napiwotzki, R., \& Reid, I. N. 1997, A\&A, 323, 819

Heger, A., \& Langer, N. 2000, ApJ, 544, 1016

Heger, A., Langer, N., \& Woosley, S. E. 2000, ApJ, 528, 368

Hillebrandt, W., \& Niemeyer, J. C. 2000, ARA\&A, 38, 191

Hiscock, W. 1998 [gr-qc/9807036]

Höflich, P., \& Khokhlov, A. 1996, ApJ, 457, 500

Höflich, P., Khokhlov, A., Wheeler, J. C., et al. 1996, ApJ, 472, L81

Höflich, P., Nomoto, K., Umeda, H., \& Wheeler, J. C. 2000, ApJ, 528, 590

Höflich, P., \& Stein, J. 2002, ApJ, 568, 779

Höflich, P., Wheeler, J. C., \& Thielemann, F. K. 1998, ApJ, 495, 617

Howell, D. A., Höflich, P., Wang, L., \& Wheeler, J. C. 2001, ApJ, 556, 302

Hubbard, W. B., \& Lampe, M. 1969, ApJS, 18, 297

Iben, I. Jr. 1982, ApJ, 259, 244

Iglesias, C. A., \& Rogers, F. J. 1996, ApJ, 464, 943

Imamura, J. N., Toman, J., Durisen, R. H., Pickett, B., \& Yang, S. 1995, ApJ, 444, 363

Itoh, N., Kohyama, Y., \& Takeuchi, H. 1987, ApJ, 317, 733

Iwamoto, K., Brachwitz, F., Nomoto, K., et al. 1999, ApJS, 125, 439

James, R. A. 1964, ApJ, 140, 552

Karino, S., \& Eriguchi, Y. 2002, ApJ, 578, 413

Kasen, D., Nugent, P., Wang, L., et al. 2003, ApJ, 593, 788

Kato, M., \& Hachisu, I. 1999, ApJ, 513, L41

Kawai, Y., Saio, H., \& Nomoto, K. 1988, ApJ, 328, 207

Kawaler, S. D. 2003, in Stellar Rotation, Proc. IAU Symp., 215, ed. A. Maeder, \& P. Eenes, in press

Kippenhahn, R., \& Möllenhoff, C. 1974, Ap. Space. Sci., 31, 117

Kippenhahn, R., \& Thomas, H.-C. 1970, in Stellar Rotation, ed. A. Slettebak, IAU Coll., 4

Kippenhahn, R., \& Thomas, H.-C. 1978, A\&A, 63, 265
Khokhlov, A. M. 1991a, A\&A, 245, 114

Khokhlov, A. M. 1991b, A\&A, 246, 383

Khokhlov, A. M., Oran, E. S., \& Wheeler, J. C. 1997, ApJ, 478, 678

Koester, D., Dreizler, S., Weidemann, V., \& Allard, N. F. 1998, A\&A, 338,617

Langer, N., Deutschmann, A., Wellstein, S., \& Höflich, P. 2000, A\&A, 362,1046

Langer, N., Heger, A., Wellstein, S., \& Herwig, F. 1999, A\&A, 346, L37

Langer, N., Kiriakidis, M., El Eid, M. F., Fricke, K. J., \& Weiss, A. 1988, A\&A, 192, 177

Langer, N., Yoon, S.-C., Petrovic, J., \& Heger, A. 2003, in Stellar Rotation, Proc. IAU Symp., 215, ed. A. Maeder, P. Eenens (San Francisco: ASP), in press [arXiv: astro-ph/0302232]

Langer, N., Yoon, S.-C., Wellstein, S., \& Scheithauer, S. 2002, ed. B. T. Gaensicke et al., ASP Conf. Proc., 261, 252

Leibundgut, B. 2000, A\&AR, 10, 179

Leibundgut, B. 2001, ARA\&A, 39, 67

Li, W., Filippenko, A. V., Chornock, R., et al. 2003, PASP, 115, 453

Li, X.-D., \& van den Heuvel, E. P. J. 1997, A\&A, 322, L9

Lindblom, L. 1999, Phys. Rev. D, 60, 4007

Lindblom, L., Tohline, J. E., \& Vallisneri, M. 2002, Phys. Rev. D, 65, 4039

Lisewski, A. M., Hillebrandt, W., \& Woosley, S. E. 2000, ApJ, 538, 831

Livio, M. 2001, in Cosmic evolution, ed. E. Vangioni, R. Ferlet, \& M. Lemoine (New Jersey: World Sceintific)

Livio, M., \& Pringle, J. E. 1998, ApJ, 505, 339

Lynden-Bell, D., \& Ostriker, J. P. 1967, MNRAS, 136, 293

Maeder, A., \& Meynet, G. 2000, ARA\&A, 38, 143

Meynet, G., \& Maeder, A. 1997, A\&A, 321, 465

Mochkovitch, R., \& Livio, M. 1989, A\&A, 209, 111

Monaghan, J. J. 1966, MNRAS, 132, 305

Nandkumar, R., \& Pethick, C. J. 1984, MNRAS, 209, 511

Narayan, R., \& Popham, R. 1989, ApJ, 346, L25

Neo, S., Miyaji, S., Nomoto, K., \& Sugimoto, D. 1977, PASJ, 29, 249

Niemeyer, J. C. 1999, ApJ, 523, L57

Niemeyer, J. C., \& Woosley, S. E. 1997, ApJ, 475, 740

Nomoto, K. 1982, ApJ, 253, 798

Nomoto, K., \& Kondo, Y. 1991, ApJ, 367, 19

Nomoto, K., Thielemann, F. K., \& Yokoi, K. 1984, ApJ, 286, 644

Nomoto, K., Uenishi, T., Kobayashi, C., et al. 2003, in From Twilights to Highlight: The Physics of Supernovae, ed. W. Hillibrandt, \& B. Leibundgut (Springer), 115

Ostriker, J. P. 1966, ApJ, 71, 394

Ostriker, J. P., \& Bodenheimer, P. 1968, ApJ, 151, 1089

Ostriker, J. P., \& Bodenheimer, P. 1973, ApJ, 180, 171

Ostriker, J. P., \& Mark, J. W.-K. 1968, ApJ, 151, 1075

Ostriker, J. P., \& Tassoul, J. L. 1969, ApJ, 155, 987

Paczyński, B. 1991, ApJ, 370, 597

Perlmutter, S., Aldering, G., Goldhaber, G., et al. 1999, ApJ, 517, 565

Phillips, M. M. 1993, ApJ, 413, L105

Piersanti, L., Cassisi, S., Iben, I. Jr., \& Tornambé, A. 2000, ApJ, 535, 932

Piersanti, L., Gagliardi, S., Iben, I. Jr., \& Tornambé, A. 2003, ApJ, 583, 885

Popham, R., \& Narayan, R. 1991, ApJ, 370, 604

Reinecke, M., Hillebrandt, W., \& Niemeyer, J. C. 2002, A\&A, 391, 1167

Riess, A. G., Filippenko, A. V., Liu, M. C., et al. 2000, ApJ, 536, 62

Ritter, H. 1985, A\&A, 148, 207

Roxburgh, I. W. 1965, Z. Astrophys., 62, 134

Shapiro, S. L., \& Teukolsky, S. A. 1983, Balack Holes, White Dwarfs and Neutron Stars (New York: Wiley) 
Saio, H., \& Nomoto, K. 1985, A\&A, 150, L21

Saio, H., \& Nomoto, K. 1998, ApJ, 500, 388

Sion, E. M. 1995, ApJ, 438, 876

Sion, E. M. 1999, PASP, 111, 532

Spitzer, L. J. 1962, Physics of fully Ionized Gases (New York: Interscience Publ.)

Spruit, H. C. 2002, A\&A, 381, 923

Townsley, D. M., \& Bildsten, L. 2002, in Physics of Cataclysmic Variables and Related Objects, ed. B. T. Gaensicke et al., ASP Conf. Proc. Ser., 261, 31

Uenishi, T., Nomoto, K., \& Hachisu, I. 2003, ApJ, 595, 1094

Umeda, H., Nomoto, K., Kobayashi, C., Hachisu, I., \& Kato, M. 1999, ApJ, 522, L43

Walleborn, J., \& Baus, M. 1978, Phys. Rev. A, 18, 1737
Wang, L., Baade, L., Höflich, P., et al. 2003, ApJ, 591, 1110

Woosley, S. E., Wunsch, S., \& Kuhlen, M. 2003

[arXiv: astro-ph/0307565]

Yoon, S.-C. 2004, Ph.D. Thesis, Utrecht University

Yoon, S.-C., \& Langer, N. 2002, in Physics of Cataclysmic Variables and Related Objects, ed. B. T. Gaensicke et al., ASP Conf. Proc. Ser., 261, 79

Yoon, S.-C., \& Langer, N. 2003, A\&A, 412, L53

Yoon, S.-C., \& Langer, N. 2004, A\&A, 419, 645

Yoon, S.-C., Langer, N., \& Scheithauer, S. 2004, A\&A, submitted

Zahn, J.-P. 1974, in Stellar Instability and Evolution, ed. P. Ledoux, A. Noels, \& A. W. Rogers (Dortrecht: Reidel), IAU Symp., 59, 185

Zahn, J.-P. 1992, A\&A, 265, 116 